\title{
Statistics of interfacial fluctuations of radially growing clusters
}

\author{
Carlos Escudero* \\ Departamento de Economía Cuantitativa \& Instituto de Ciencias Matemáticas (CSIC-UAM-UC3M-UCM), \\ Universidad Autónoma de Madrid, Ciudad Universitaria de Cantoblanco, E-28049 Madrid, Spain \\ (Received 18 January 2011; revised manuscript received 12 April 2011; published 28 September 2011)
}

\begin{abstract}
The dynamics of fluctuating radially growing interfaces is approached using the formalism of stochastic growth equations on growing domains. This framework reveals a number of dynamic features arising during surface growth. For fast growth, dilution, which spatially reorders the incoming matter, is responsible for the transmission of correlations. Its effects include the erasing of memory with respect to the initial condition, a partial attenuation of geometrically originated instabilities, and the restoration of universality in some special cases in which the critical exponents depend on the parameters of the equation of motion. In this sense, dilution rends the dynamics more similar to the usual one of planar systems. This fast growth regime is also characterized by the spatial decorrelation of the interface, which, in the case of radially growing interfaces, naturally originates rapid roughening and scale-dependent fractality, and suggests the advent of a self-similar fractal dimension. The center-of-mass fluctuations of growing clusters are also studied, and our analysis suggests the possible nonapplicability of usual scalings to the long-range surface fluctuations of the radial Eden model. In fact, our study points to the fact that this model belongs to a dilution-free universality class.
\end{abstract}

DOI: 10.1103/PhysRevE.84.031131

PACS number(s): 05.40.-a, 89.75.-k, 05.10.Gg, 64.60.Ht

\section{INTRODUCTION}

The study of fluctuating interfaces has occupied an important place within statistical mechanics in recent and not so recent times. The origins of this interest are practical, due to the vast range of potential applications that this theory may have, and theoretical, as some of the universality classes discovered within this framework are claimed to play an important role in other areas of physics [1]. While the great majority of works on this topic has concentrated on strip or slab geometries, it is true that at the very beginning of the theoretical studies on nonequilibrium growth, one finds the seminal works by Eden, focused on radial shapes [2,3]. To a certain extent, the motivation of considering radial forms is related to biological growth, as, for instance, the Eden model can be thought of as a simplified description of a developing cell colony. The Eden and other related discrete models have been computationally analyzed through the years, and the results obtained have been put in the context of stochastic growth theory (see, e.g., [4] and references therein). One of the most important models of nonequilibrium growth of radial systems is diffusion-limited aggregation (DLA) [5]. It is related to physical phenomena such as electrodeposition, Hele-Shaw flow, mineral deposits, and dielectric breakdown. The theory we present in this paper is not in a state to be able to describe such complex branched structures. Nevertheless, as we see in the following, it might be related to specific limits of DLA processes.

Apart from the interest in modeling, there is a genuine theoretical motivation in understanding the dynamics of growing radial clusters. The Eden model is actually a sort of first-passage percolation [6], and the scaling limit of percolation models has been studied by means of field-theoretic approaches [7] and stochastic processes like SchrammLoewner evolution [8]. A natural theoretical question to be

*cel@icmat.es answered is in which cases the Family-Vicsek scaling [9], basic for describing planar growth processes, is able to capture the behavior of surface fluctuations of growing radial clusters.

The use of stochastic differential equations, very widespread in the modeling of planar growth profiles, has been not as commonly employed in the case of radial growth. A series of works constitutes an exception to this rule [10-16], as they proposed a partial differential equation with stochastic terms as a benchmark for analyzing the dynamics of radial interfaces. Because studying this sort of equations is complicated by the nonlinearities implied by reparametrization invariance, a simplified version in which only the substrate growth was considered was introduced in [17]. Already in this case it was apparent that for rapidly growing interface dilution, which is responsible for matter redistribution as the substrate grows [18], propagates the correlations when large spatiotemporal scales are considered. It is also capable of erasing the memory effects that would otherwise arise; let us show how. In [17] we considered the linear equation for stochastic growth on a growing domain,

$$
\partial_{t} h=-D\left(\frac{t_{0}}{t}\right)^{\zeta \gamma}|\nabla|^{\zeta} h-\frac{d \gamma}{t} h+\gamma F t^{\gamma-1}+\left(\frac{t_{0}}{t}\right)^{d \gamma / 2} \xi(x, t),
$$

where the domain grows following the power law $t^{\gamma}, \gamma>0$ is the growth index, and $-(d \gamma / t) h$ is the term taking into account dilution [17]. Dilution refers to the fact that the interfacial matter, as the interface grows, becomes redistributed in a larger domain. Its Fourier transformed version, for $n \geqslant 1$, is

$$
\frac{d h_{n}}{d t}=-D\left(\frac{t_{0}}{t}\right)^{\zeta \gamma} \frac{\pi^{\zeta}|n|^{\zeta}}{L_{0}^{\zeta}} h_{n}-\frac{d \gamma}{t} h_{n}+\left(\frac{t_{0}}{t}\right)^{d \gamma / 2} \xi_{n}(t) .
$$


This equation can be readily solved for $\gamma>1 / \zeta$ and in the long time limit,

$$
\begin{aligned}
h_{n}(t)= & \left(t / t_{0}\right)^{-d \gamma} \exp \left[\frac{D t_{0}}{1-\zeta \gamma} \frac{\pi^{\zeta}|n|^{\zeta}}{L_{0}^{\zeta}}\right] h_{n}\left(t_{0}\right) \\
& +\left(t / t_{0}\right)^{-d \gamma} \int_{t_{0}}^{t}\left(\frac{\tau}{t_{0}}\right)^{d \gamma / 2} \xi_{n}(\tau) d \tau,
\end{aligned}
$$

and so the dependence on the initial condition tends to 0 as a power law for long times. This is, as mentioned, one of the consequences of dilution. If we considered the dilatation transformation $x \rightarrow\left(t / t_{0}\right)^{\gamma} x$, we would again find Eq. (1), but this time without the dilution term. This corresponds to a dilatation of mass and space simultaneously. The solution now becomes

$$
\begin{aligned}
h_{n}(t)= & \exp \left[\frac{D t_{0}}{1-\zeta \gamma} \frac{\pi^{\zeta}|n|^{\zeta}}{L_{0}^{\zeta}}\right] h_{n}\left(t_{0}\right) \\
& +\int_{t_{0}}^{t}\left(\frac{t_{0}}{\tau}\right)^{d \gamma / 2} \xi_{n}(\tau) d \tau,
\end{aligned}
$$

and so the dependence on the initial condition remains for all times. In the first case the long-time solution becomes spatially uncorrelated, and in the second one, only part of the initial correlations survive. As an abuse of language, we talk about decorrelation in both cases. The memory effects that affect the solution in the no-dilution (or dilatation) situation separate its behavior from the one dictated by the Family-Vicsek scaling [17,19]. For $\gamma<1 / \zeta$ the memory effects and the corresponding dependence on the initial condition disappear exponentially rapidly for long times as a consequence of the effect of diffusion.

We start discussing dilution as the mechanism that controls the amount of matter on the interface. Pure diffusion on a growing domain is described by the equation

$$
\partial_{t} h=D\left(\frac{t_{0}}{t}\right)^{2 \gamma} \nabla^{2} h-\frac{d \gamma}{t} h,
$$

in Eulerian coordinates $x \in\left[0, L_{0}\right] \times \cdots \times\left[0, L_{0}\right]$ (see [17]), and where dilution has been taken into account. The total mass on the surface is conserved,

$$
\begin{aligned}
\int_{0}^{L(t)} \cdots \int_{0}^{L(t)} h(y, t) d y & =\left(\frac{t}{t_{0}}\right)^{d \gamma} \int_{0}^{L_{0}} \cdots \int_{0}^{L_{0}} h(x, t) d x \\
& =\int_{0}^{L_{0}} \cdots \int_{0}^{L_{0}} h\left(x, t_{0}\right) d x
\end{aligned}
$$

where $y \equiv\left[L(t) / L_{0}\right] x$ denotes the set of Lagrangian coordinates. Note that we are using the shorthand notations $x=$ $\left(x_{1}, \ldots, x_{d}\right), y=\left(y_{1}, \ldots, y_{d}\right), d x=d x_{1} \cdots d x_{d}$, and $d y=$ $d y_{1} \cdots d y_{d}$. In the no-dilution situation we find

$$
\begin{aligned}
\int_{0}^{L(t)} \cdots \int_{0}^{L(t)} h(y, t) d y & =\left(\frac{t}{t_{0}}\right)^{d \gamma} \int_{0}^{L_{0}} \cdots \int_{0}^{L_{0}} h(x, t) d x \\
& =\left(\frac{t}{t_{0}}\right)^{d \gamma} \int_{0}^{L_{0}} \cdots \int_{0}^{L_{0}} h\left(x, t_{0}\right) d x .
\end{aligned}
$$

This second case is pure dilatation, which implies not only that the space grows, but also that the interfacial matter grows at the same rate, in such a way that the average density remains constant. Note that this process of matter dilatation, as well as the spatial growth, is a deterministic process. These calculations show that both dilution and dilatation dynamics are physically motivated and have a number of measurable differences. It is worth remarking here that all previous works except [17] and [20] have exclusively considered dilatation dynamics. Even in the different field of reaction-diffusion dynamics in which the dilution term was derived, the focus was on the limit in which it was irrelevant [18].

This work is devoted to exploring further the consequences of dilution, dilatation, and decorrelation and their effects on scaling of radial interfaces. We use, in some cases, radial stochastic growth equations, which may show up instabilities [16], and explore the interplay of dilution with them. In other cases, when instabilities do not play a determinant role and for the sake of simplicity, we consider stochastic growth equations on growing domains. The outline of the paper is as follows: In Sec. II we consider the simplest radial growth process, radial random deposition, and derive for the first time the two-point space-time correlation functions. In Sec. III and $\mathrm{Sec}$. IV we compute for the first time the two-point space-time correlators for stochastic growth equations, taking into account simultaneously random deposition and diffusion in the absence and presence of instabilities, respectively. In Sec. V and Sec. VI we show, for the first time, that radial stochastic growth equations give rise naturally to the phenomena of rapid roughening and scale-dependent fractality of surfaces. In Sec. VII we mention some of the problems that arise when studying the radial counterpart of nonlinear stochastic growth equations and in Sec. VIII we calculate for the first time the center-of-mass fluctuations of the cluster interfaces described by radial stochastic growth equations. Finally, in Sec. IX we apply our results to Eden clusters and in Sec. X we draw our main conclusions.

\section{RADIAL RANDOM DEPOSITION}

In this section we construct for the first time two-point space-time correlation functions for the radial random deposition process. This quantities are fundamental in order to statistically characterize fluctuating interfaces.

In order to construct radial growth equations one may invoke the reparametrization invariance principle [21,22], as has already been done a number of times $[10,11,13-16]$. In the case of white and Gaussian fluctuations, the $d$-dimensional spherical noise is given by

$$
\begin{aligned}
& \frac{1}{\sqrt{g[\vec{\theta}, r(\vec{\theta}, t)]}} \xi(\vec{\theta}, t), \quad\langle\xi(\vec{\theta}, t)\rangle=0, \\
& \langle\xi(\vec{\theta}, t) \xi(\vec{\theta}, t)\rangle=\epsilon \delta\left(\vec{\theta}-\vec{\theta}^{\prime}\right) \delta\left(t-t^{\prime}\right),
\end{aligned}
$$

where $g=\operatorname{det}\left(g_{i j}\right)=\operatorname{det}\left(\partial_{i} \vec{r} \cdot \partial_{j} \vec{r}\right)$ is the determinant of the metric tensor. Under the small gradient assumption $\left|\nabla_{\vec{\theta}} r\right| \ll$ $r$, one finds $g \approx \mathcal{J}(r, \vec{\theta})^{2}$, where $\mathcal{J}$ is the Jacobian determinant of the change of variables from the Cartesian representation $(\vec{x}, h)$ to the polar representation $(\vec{\theta}, r)$. We also have the factorization $\mathcal{J}(r, \vec{\theta})^{2}=r^{2 d} J(\vec{\theta})^{2}$, where $J$ is the Jacobian evaluated at $r=1$. 
The simplest growth process is possibly the radial random deposition model. If the growth rate is explicitly time dependent, then the growth equation reads

$$
\partial_{t} r=F \gamma t^{\gamma-1}+\frac{1}{r^{d / 2} J(\vec{\theta})^{1 / 2}} \xi(\vec{\theta}, t),
$$

in the absence of dilution. Here $r(\vec{\theta}, t)$ is the value of the radius at the angular position $\vec{\theta}$ and time $t, F>0$ is the constant prefactor of the growth rate, $\gamma>0$ is the growth index, $d$ is the number of angles used to parametrize the cluster surface (so the cluster grows in $d+1$ spatial dimensions), and $\xi$ is a zero-mean Gaussian noise, whose correlation is given by

$$
\left\langle\xi(\vec{\theta}, t) \xi\left(\vec{\theta}^{\prime}, s\right)\right\rangle=\epsilon \delta\left(\vec{\theta}-\vec{\theta}^{\prime}\right) \delta(t-s) .
$$

The equation for the first moment can be easily obtained,

$$
\partial_{t}\langle r\rangle=F \gamma t^{\gamma-1},
$$

due to the Itô interpretation of the noise, and we can integrate it to get

$$
\langle r(\vec{\theta}, t)\rangle=F t^{\gamma},
$$

where we have assumed the radially symmetric initial condition $r\left(\vec{\theta}, t_{0}\right)=F t_{0}^{\gamma}$ and $t_{0} \leqslant t$ is the absolute origin of time. It is difficult to obtain more information from the full equation, (10), so we perform a perturbative expansion. We assume the solution form

$$
r(\vec{\theta}, t)=R(t)+\sqrt{\epsilon} \rho_{1}(\vec{\theta}, t),
$$

where the noise intensity $\epsilon$ is used as the small parameter [23]. Substituting this solution form into Eq. (10) we obtain the equations

$$
\begin{gathered}
\partial_{t} R=F \gamma t^{\gamma-1}, \\
\partial_{t} \rho_{1}=\frac{1}{F^{d / 2} t^{\gamma d / 2}} \frac{\eta(\vec{\theta}, t)}{J(\vec{\theta})^{1 / 2}},
\end{gathered}
$$

where $\xi=\sqrt{\epsilon} \eta$. These equations have been derived assuming $\sqrt{\epsilon} \ll F t^{\gamma}$, a condition much more favorable (the better the larger $\gamma$ is) than the usual time-independent ones supporting small noise expansions [23]. The solution to these equations can be readily computed,

$$
\begin{aligned}
R(\vec{\theta}, t)=F t^{\gamma} & \left\langle\rho_{1}(\vec{\theta}, t)\right\rangle=0 \\
\left\langle\rho_{1}(\vec{\theta}, t) \rho_{1}\left(\vec{\theta}^{\prime}, s\right)\right\rangle= & \frac{F^{-d}}{1-\gamma d}\left[(\min \{t, s\})^{1-\gamma d}-t_{0}^{1-\gamma d}\right] \\
& \times \frac{\delta\left(\vec{\theta}-\vec{\theta}^{\prime}\right)}{J(\vec{\theta})}
\end{aligned}
$$

if $\gamma d \neq 1$ and where we have assumed a 0 value for the initial perturbation. If $\gamma d=1$, the correlation becomes

$$
\left\langle\rho_{1}(\vec{\theta}, t) \rho_{1}\left(\vec{\theta}^{\prime}, s\right)\right\rangle=\frac{1}{F^{d}} \ln \left[\frac{\min \{t, s\}}{t_{0}}\right] \frac{\delta\left(\vec{\theta}-\vec{\theta}^{\prime}\right)}{J(\vec{\theta})} .
$$

Here $R$ is a deterministic function and $\rho_{1}$ is a zero-mean Gaussian stochastic process that is completely determined by the correlations given above. The long-time behavior of the correlations, given by the condition $t, s \gg t_{0}$, is specified by the two-times and one-time correlation functions

$$
\begin{array}{r}
\left\langle\rho_{1}(\vec{\theta}, t) \rho_{1}\left(\vec{\theta}^{\prime}, s\right)\right\rangle=\frac{F^{-d}}{1-\gamma d}(\min \{t, s\})^{1-\gamma d} \frac{\delta\left(\vec{\theta}-\vec{\theta}^{\prime}\right)}{J(\vec{\theta})} \\
\left\langle\rho_{1}(\vec{\theta}, t) \rho_{1}\left(\vec{\theta}^{\prime}, t\right)\right\rangle=\frac{F^{-d}}{1-\gamma d} t^{1-\gamma d} \frac{\delta\left(\vec{\theta}-\vec{\theta}^{\prime}\right)}{J(\vec{\theta})}
\end{array}
$$

if $\gamma d>1$,

$$
\begin{array}{r}
\left\langle\rho_{1}(\vec{\theta}, t) \rho_{1}\left(\vec{\theta}^{\prime}, s\right)\right\rangle=\frac{1}{F^{d}} \ln (\min \{t, s\}) \frac{\delta\left(\vec{\theta}-\vec{\theta}^{\prime}\right)}{J(\vec{\theta})}, \\
\left\langle\rho_{1}(\vec{\theta}, t) \rho_{1}\left(\vec{\theta}^{\prime}, t\right)\right\rangle=\frac{1}{F^{d}} \ln (t) \frac{\delta\left(\vec{\theta}-\vec{\theta}^{\prime}\right)}{J(\vec{\theta})}
\end{array}
$$

if $\gamma d=1$, and, finally,

$$
\left\langle\rho_{1}(\vec{\theta}, t) \rho_{1}\left(\vec{\theta}^{\prime}, s\right)\right\rangle=\frac{F^{-d}}{\gamma d-1} t_{0}^{1-\gamma d} \frac{\delta\left(\vec{\theta}-\vec{\theta}^{\prime}\right)}{J(\vec{\theta})}
$$

when $\gamma d>1$. In this last case the correlation vanishes in the limit $t_{0} \rightarrow \infty$. Note that the reparametrization invariance principle is not able to capture dilution effects and it reproduces pure dilatation dynamics.

In order to introduce dilution in the radial case we may use the following functional definition, which transforms Eq. (10) into

$$
\partial_{t} r=F \gamma t^{\gamma-1}-\frac{\gamma d}{t} r+\frac{1}{r^{d / 2}} \frac{\xi(\vec{\theta}, t)}{J(\vec{\theta})^{1 / 2}},
$$

whose first moment can be exactly calculated, again taking advantage of the Itô interpretation of the noise term, yielding

$$
\langle r(\vec{\theta}, t)\rangle=\frac{F}{d+1} t^{\gamma} .
$$

Performing, as in the former case, the small noise expansion $r=R+\sqrt{\epsilon} \rho_{1}$, we again find $R=\langle r\rangle$. The perturbation obeys the equation

$$
\partial_{t} \rho_{1}=-\frac{\gamma d}{t} \rho_{1}+\frac{(d+1)^{d / 2}}{F^{d / 2} t^{\gamma d / 2}} \frac{\eta(\vec{\theta}, t)}{J(\vec{\theta})^{1 / 2}},
$$

and so the perturbation has zero mean and its long-time correlation is given by

$$
\begin{aligned}
\left\langle\rho_{1}(\vec{\theta}, t) \rho_{1}\left(\vec{\theta}^{\prime}, s\right)\right\rangle= & \frac{(d+1)^{d}}{F^{d}(\gamma d+1)} \min \{s, t\} \max \{s, t\}^{-\gamma d} \\
& \times \frac{\delta\left(\vec{\theta}-\vec{\theta}^{\prime}\right)}{J(\vec{\theta})}
\end{aligned}
$$

a result that holds uniformly in $\gamma$. Note that the structure of the temporal correlation is different when the effect of dilution is considered and when it is not for all $\gamma>0$. For instance, the characteristic length scale corresponding to a given angular difference is $\mathfrak{l}=\max \{s, t\}^{\gamma}\left|\vec{\theta}-\vec{\theta}^{\prime}\right|$ when dilution is present and $\mathfrak{l}=\min \{s, t\}^{\gamma}\left|\vec{\theta}-\vec{\theta}^{\prime}\right|$ in the absence of dilution. One already sees in this example that the lack of dilution causes the appearance of memory effects on the growth dynamics. The first-order correction in the small noise expansion $\rho_{1}$ is always a Gaussian stochastic process; an attempt to go beyond 


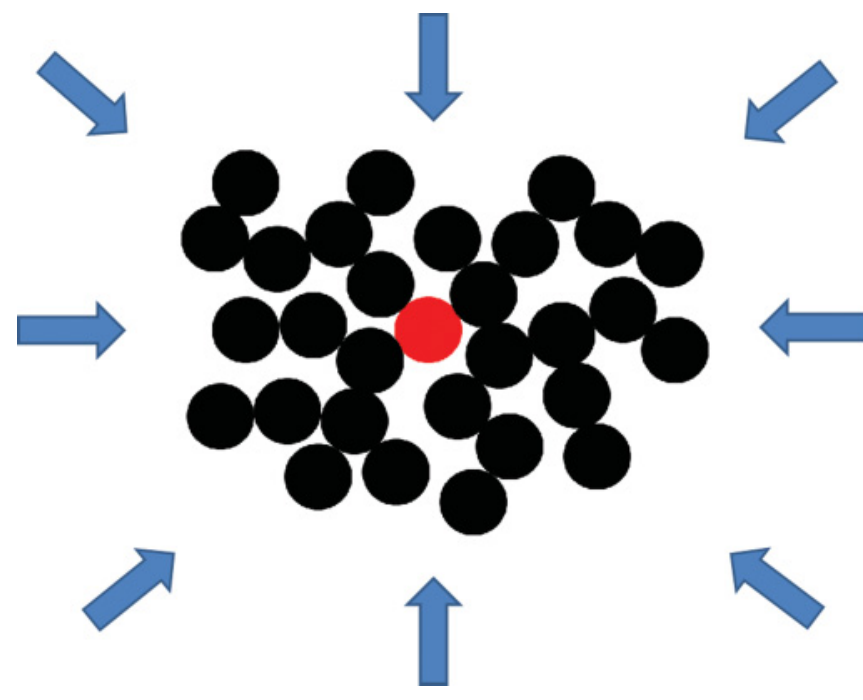

FIG. 1. (Color online) Possible realization of the random deposition process as explained in the text. New particles approach the origin [which is coincident with the center of the red (gray) particle] following a random direction. Once these particles touch another particle in the cluster, they become permanently attached to that position.

Gaussianity by deriving the second-order correction is reported in the Appendix.

One may wonder about different possible realizations of the radial random deposition process we have introduced by means of stochastic differential equations. It is of particular usefulness within the realm of statistical physics in the design of suitable discrete models. One possibility would be the proposition sketched in Fig. 1, which focuses on a two-dimensional system. We consider an off-lattice model to get rid of possible undesirable anisotropy effects which may arise when an underlying lattice is present. We start placing the center of a spherical particle at the origin; this is the seed particle shown in red (gray) in Fig. 1. The next step is choosing a random number uniformly distributed in the interval $[0,2 \pi)$. This number selects the angle which marks the direction along which a new particle (identical to the seed one) travels from a long distance (much longer than the cluster radius) toward the origin. This particle does not deviate in any sense from this direction until it touches the seed particle, to which it becomes permanently attached. The process is now repeated: a new angle is randomly selected and a new particle follows the corresponding direction until it touches any other particle in the system; in this instant it again becomes permanently attached to that position. Iterating this process we arrive at a growing cluster like the one depicted in Fig. 1. This sort of cluster, in the limit in which the cluster radius is much longer than the particles radius, constitutes a possible realization of the radial random deposition process. The generalization to arbitrary dimensions is straightforward. In the case of a $d+1$-dimensional cluster the direction along which a new particle approaches the growing cluster is selected by $d$ random angles; $d-1$ of them (the polar angles) are uniformly distributed in $[0, \pi]$, and the $d$ th angle (the azimuth angle) is uniformly distributed in $[0,2 \pi)$. The dynamics of the process is otherwise identical. We note that this discrete model can be understood as a particular limit of a DLA process in which diffusion is substituted by a random drift.

Radial random deposition models are important because they act as statistical attractors of the solutions to different radial growth equations. Indeed, if the growth index is high enough, then the long-time large-scale properties of the solutions to different radial equations approach the corresponding properties of the solutions to the radial random deposition equations. This has been characterized as the decorrelation limit in [17]. It is thus interesting to visualize the solutions to these equations. This is carried out in Fig. 2. In this figure we represent the function $r(\vec{\theta}, t)$ from Eq. (14) for four different values of $\gamma$ and for $d=1$. The result is four clearly different morphologies, which become more similar to the radially symmetric deterministic growth process $R(t)$ for larger $\gamma$.

\section{RANDOM DEPOSITION AND DIFFUSION}

Our next step, in order to approach more complex and realistic growth processes, is to add diffusion to a random deposition equation of growth. This sort of equation may be derived using reparametrization invariance as in [16]. Following this reference and the former section, we perform a small noise expansion and concentrate on the equation for the Gaussian perturbation. Again, our goal will be deriving for the first time two-point space-time correlation functions for this type of processes. In this section we consider a number of cases which do not show instabilities, and the study of these is postponed to the following section. The equation for the perturbation in $d=1$ is [16]

$$
\partial_{t} \rho=\frac{D_{\zeta}}{\left(F t^{\gamma}\right)^{\zeta}} \Lambda_{\theta}^{\zeta} \rho+\frac{1}{\sqrt{F t^{\gamma}}} \eta(\theta, t),
$$

where $\Lambda_{\theta}^{\zeta}$ is a fractional differential operator of order $\zeta$, and dilution has not been considered. The dynamics for $\zeta>d$, which in turn implies that in the linear case the growth exponent $\beta>0$ and the interface is consequently rough, has been considered in [17]; herein we move to studying the marginal case $\zeta=d$, which turns out to have interesting properties. The case $\zeta<d$ is not as interesting, as it corresponds to flat interfaces; an analogous calculation to the corresponding one in [16] for $\gamma=1$ and $\zeta<1$ shows that

$$
\left\langle\rho(\theta, t) \rho\left(\theta^{\prime}, s\right)\right\rangle \rightarrow 0 \quad \text { when } \quad t, s \rightarrow \infty,
$$

independently of the value of $t_{0}$.

$$
\text { If } \zeta=\gamma=1 \text {, the correlation reads }
$$

$$
\begin{aligned}
& \left\langle\rho(\theta, t) \rho\left(\theta^{\prime}, s\right)\right\rangle \\
& \quad=\frac{1}{4 \pi D} \ln \left[\frac{(t s)^{D / F}}{(s / t)^{D / F}+(t / s)^{D / F}-2 \cos \left(\theta-\theta^{\prime}\right)}\right] .
\end{aligned}
$$

The one-time correlation adopts the form

$$
\left\langle\rho(\theta, t) \rho\left(\theta^{\prime}, t\right)\right\rangle=\frac{1}{4 \pi D} \ln \left[\frac{t^{2 D / F}}{2-2 \cos \left(\theta-\theta^{\prime}\right)}\right],
$$

which reduces to

$$
\left\langle\rho(\theta, t) \rho\left(\theta^{\prime}, t\right)\right\rangle \approx \frac{1}{2 \pi F} \ln \left(\frac{t}{\left|\theta-\theta^{\prime}\right|^{F / D}}\right),
$$




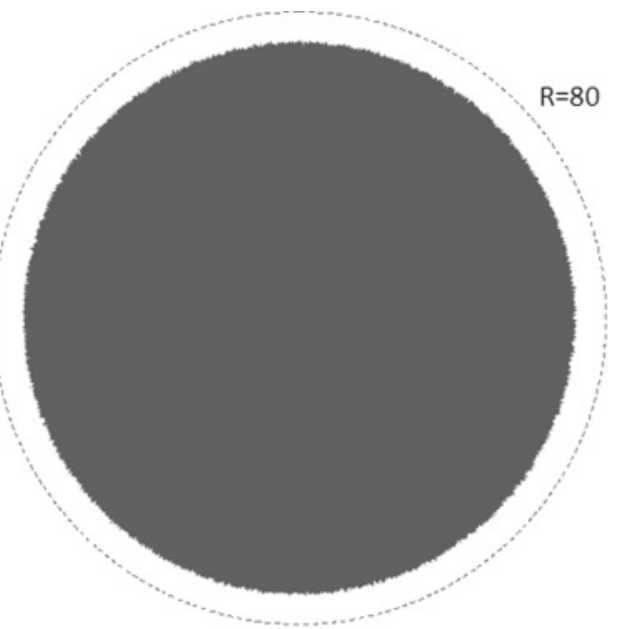

(a)

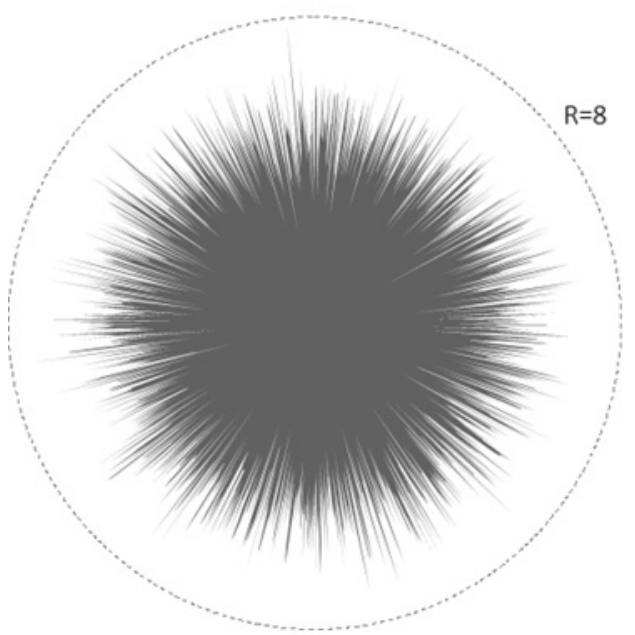

(c)

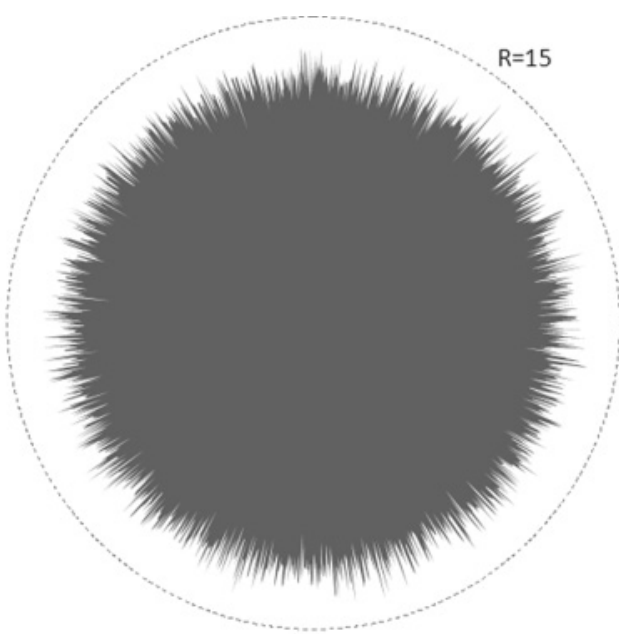

(b)

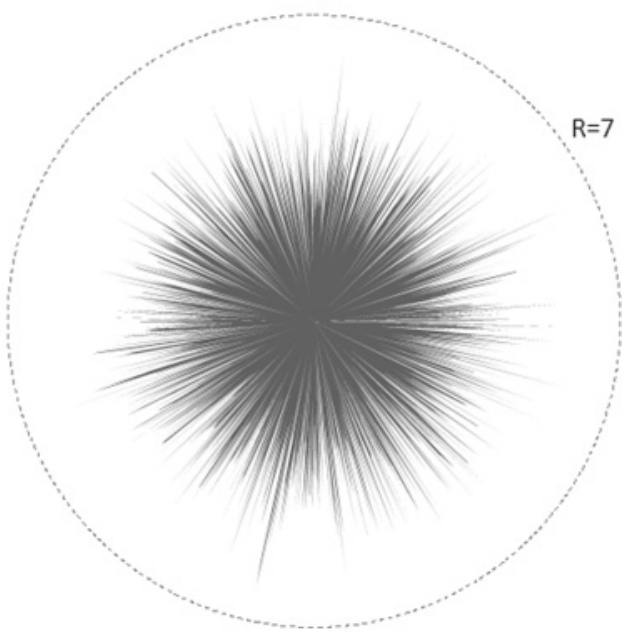

(d)

FIG. 2. Radial random deposition growth processes plotted at $t=6$. The values of the parameters are $F=2, \epsilon=(200 \pi)^{-1}$, and the system has been discretized in $2^{11}$ spatial points. We have used as the initial condition $r(\theta, 1)=2$ (note that we have used as the initial time $t_{0}=1$ ). The outer, dashed circumferences give an estimate of the size of the cluster (the corresponding values of the radius are indicated in each panel). (a) $\gamma=2$. (b) $\gamma=1$. (c) $\gamma=1 / 2$. (d) $\gamma=1 / 4$.

when we consider local in space dynamics, that is, in the limit $\theta \approx \theta^{\prime}$. Note that this result allows us to define the local dynamic exponent $z_{\text {loc }}=F / D \in(0, \infty)$, which depends continuously on the equation parameters $F$ and $D$ and, thus, is nonuniversal, as we noted in [16]. In terms of the arc-length variable $\ell-\ell^{\prime}=t\left(\theta-\theta^{\prime}\right)$, we find

$$
\left\langle\rho(\ell, t) \rho\left(\ell^{\prime}, t\right)\right\rangle \approx \frac{F^{-1}+D^{-1}}{2 \pi} \ln \left(\frac{t}{\left|\ell-\ell^{\prime}\right|^{F /(D+F)}}\right),
$$

where the dynamical exponent in terms of the arc-length variable $z_{\ell}=F /(D+F) \in(0,1)$ is again nonuniversal. If we take dilution into account, Eq. (30) transforms to

$$
\partial_{t} \rho=\frac{D}{F t} \Lambda_{\theta} \rho-\frac{1}{t} \rho+\frac{1}{\sqrt{F t}} \eta(\theta, t) .
$$

The solution has zero mean and its correlation is given by

$$
\begin{aligned}
& \left\langle\rho(\theta, t) \rho\left(\theta^{\prime}, s\right)\right\rangle \\
& =\frac{\min \{s, t\} / \max \{s, t\}}{4 \pi F}+\frac{(\min \{s, t\} / \max \{s, t\})^{1+D / F}}{2 \pi(F+D)}
\end{aligned}
$$

$$
\begin{aligned}
& \times \operatorname{Re}\left\{e ^ { i ( \theta - \theta ^ { \prime } ) } { } _ { 2 } F _ { 1 } \left[1,1+\frac{F}{D} ; 2\right.\right. \\
& \left.\left.+\frac{F}{D} ; e^{i\left(\theta-\theta^{\prime}\right)}\left(\frac{\min \{s, t\}}{\max \{s, t\}}\right)^{D / F}\right]\right\},
\end{aligned}
$$

where $\operatorname{Re}(\cdot)$ denotes the real part and ${ }_{2} F_{1}(\cdot, \cdot ; \cdot ; \cdot)$ is the Gauss hypergeometric function [24]. This correlation, for $s=t$ and for small angular scales $\theta \approx \theta^{\prime}$, becomes, at leading order,

$$
\left\langle\rho(\theta, t) \rho\left(\theta^{\prime}, t\right)\right\rangle \approx \frac{-1}{2 \pi D} \ln \left(\left|\theta-\theta^{\prime}\right|\right),
$$

which is time independent, and for the arc-length variable,

$$
\left\langle\rho(\ell, t) \rho\left(\ell^{\prime}, t\right)\right\rangle \approx \frac{1}{2 \pi D} \ln \left(\frac{t}{\left|\ell-\ell^{\prime}\right|}\right),
$$

for which the planar scaling and the universal dynamical exponent $z=1$ are recovered; see Eq. (C5) in [16]. This is yet another example, this time of a different nature, of how dilution is able to restore the Family-Vicsek scaling $[17,19]$. 
If $\zeta=1$ and $\gamma<1$, we find the following correlation function:

$$
\begin{aligned}
\left\langle\rho(\theta, t) \rho\left(\theta^{\prime}, s\right)\right\rangle= & \frac{[\min \{t, s\}]^{1-\gamma}}{2 \pi F(1-\gamma)}-\frac{1}{4 \pi D} \ln \{1 \\
& +\exp \left[-\frac{2 D}{F(1-\gamma)}\left|t^{1-\gamma}-s^{1-\gamma}\right|\right] \\
& -2 \exp \left[-\frac{D}{F(1-\gamma)}\left|t^{1-\gamma}-s^{1-\gamma}\right|\right] \\
& \left.\times \cos \left(\theta-\theta^{\prime}\right)\right\} .
\end{aligned}
$$

When $t=s$ we get

$$
\begin{aligned}
& \left\langle\rho(\theta, t) \rho\left(\theta^{\prime}, t\right)\right\rangle \\
& \quad=\frac{t^{1-\gamma}}{2 \pi F(1-\gamma)}-\frac{1}{4 \pi D} \ln \left[2-2 \cos \left(\theta-\theta^{\prime}\right)\right],
\end{aligned}
$$

and considering local spatial dynamics we arrive at

$$
\begin{aligned}
\left\langle\rho(\theta, t) \rho\left(\theta^{\prime}, t\right)\right\rangle & \approx \frac{t^{1-\gamma}}{2 \pi F(1-\gamma)}-\frac{1}{2 \pi D} \ln \left(\left|\theta-\theta^{\prime}\right|\right) \\
& =\frac{1}{2 \pi F(1-\gamma)} \ln \left[\frac{e^{t^{1-\gamma}}}{\left|\theta-\theta^{\prime}\right|^{F(1-\gamma) / D}}\right],
\end{aligned}
$$

an expression that does not allow definition of a local dynamic exponent, or, alternatively, $z_{\text {loc }}=0$, due to the exponentially fast spreading of the correlations. These last three expressions contain two clearly different terms. The first one is the zeroth mode component of the correlation, which does not achieve long-time saturation. The second term is the nontrivial stationary part of the correlation generated along the evolution. As can be seen, both spatial and temporal correlations are generated.

When the dilution term is taken into account we find the correlation

$$
\begin{aligned}
\left\langle\rho(\theta, t) \rho\left(\theta^{\prime}, s\right)\right\rangle= & \frac{\min \{t, s\}[\max \{t, s\}]^{-\gamma}}{2 \pi F(\gamma+1)}-\frac{1}{4 \pi D} \\
& \times \ln \left\{1+\exp \left[-\frac{2 D}{F(1-\gamma)}\left|t^{1-\gamma}-s^{1-\gamma}\right|\right]\right. \\
& -2 \exp \left[-\frac{D}{F(1-\gamma)}\left|t^{1-\gamma}-s^{1-\gamma}\right|\right] \\
& \left.\times \cos \left(\theta-\theta^{\prime}\right)\right\} .
\end{aligned}
$$

When $t=s$ we get

$$
\begin{aligned}
\left\langle\rho(\theta, t) \rho\left(\theta^{\prime}, t\right)\right\rangle= & \frac{t^{1-\gamma}}{2 \pi F(\gamma+1)}-\frac{1}{4 \pi D} \\
& \times \ln \left[2-2 \cos \left(\theta-\theta^{\prime}\right)\right],
\end{aligned}
$$

and considering local spatial dynamics we arrive at

$$
\begin{aligned}
\left\langle\rho(\theta, t) \rho\left(\theta^{\prime}, t\right)\right\rangle & \approx \frac{t^{1-\gamma}}{2 \pi F(\gamma+1)}-\frac{1}{2 \pi D} \ln \left(\left|\theta-\theta^{\prime}\right|\right) \\
& =\frac{1}{2 \pi F(\gamma+1)} \ln \left[\frac{e^{t^{1-\gamma}}}{\left|\theta-\theta^{\prime}\right|^{F(\gamma+1) / D}}\right],
\end{aligned}
$$

and we see that, as in the former case, both prefactor and exponent are modified, but the still exponentially fast propagation of correlations implies an effective local dynamical exponent $z_{\text {loc }}=0$. Note that for $\gamma>1$ a radial random deposition behavior for large spatial scales is recovered.

Now we move onto the two-dimensional setting. As in the one-dimensional case we focus on the marginal situation $d=\zeta=2$, which leads us to denominate this sort of equations as spherical Edwards-Wilkinson (EW) equations, and $0<$ $\gamma \leqslant 1 / 2$, as greater values of the growth index again lead to decorrelation. The straightforward generalization of Eq. (30) is

$$
\partial_{t} \rho=\frac{K}{\left(F t^{\gamma}\right)^{2}} \nabla^{2} \rho+\frac{1}{F t^{\gamma} \sqrt{\sin (\theta)}} \eta(\theta, \phi, t),
$$

where the noise is a Gaussian random variable of zero mean and correlation given by

$$
\left\langle\xi(\theta, \phi, t) \xi\left(\theta^{\prime}, \phi^{\prime}, s\right)\right\rangle=\delta\left(\theta-\theta^{\prime}\right) \delta\left(\phi-\phi^{\prime}\right) \delta(t-s) .
$$

In this case, if $\gamma<1 / 2$, the random variable $\rho$ is a zero-mean Gaussian process whose correlation is given by

$$
\begin{aligned}
\left\langle\rho(\theta, \phi, t) \rho\left(\theta^{\prime}, \phi^{\prime}, s\right)\right\rangle= & \frac{[\min (t, s)]^{1-2 \gamma}}{4 \pi F^{2}(1-2 \gamma)}+\sum_{l=1}^{\infty} \sum_{m=-l}^{l} \frac{(-1)^{m}}{2 K\left(l+l^{2}\right)} \\
& \times \exp \left[-\frac{K\left(l+l^{2}\right)}{F^{2}(1-2 \gamma)}\left|t^{1-2 \gamma}-s^{1-2 \gamma}\right|\right] \\
& \times Y_{-m}^{l}(\theta, \phi) Y_{m}^{l}\left(\theta^{\prime}, \phi^{\prime}\right),
\end{aligned}
$$

where the expansion has been performed on the spherical harmonics basis $Y_{m}^{l}(\theta, \phi)$. If $\gamma=1 / 2$, then $\rho$ becomes a zero-mean Gaussian random variable with the new correlation

$$
\begin{aligned}
\left\langle\rho(\theta, \phi, t) \rho\left(\theta^{\prime}, \phi^{\prime}, s\right)\right\rangle= & \frac{\ln [\min (t, s)]}{4 \pi F^{2}}+\sum_{l=1}^{\infty} \sum_{m=-l}^{l} \frac{(-1)^{m}}{2 K\left(l+l^{2}\right)} \\
& \times\left[\frac{\min (s, t)}{\max (s, t)}\right]^{K\left(l+l^{2}\right) / F^{2}} \\
& \times Y_{-m}^{l}(\theta, \phi) Y_{m}^{l}\left(\theta^{\prime}, \phi^{\prime}\right) .
\end{aligned}
$$

It is clear that these correlations are again composed of two different terms; the first one, associated with the $l=0$ mode, never saturates, and the second one, associated with the rest of modes $l>0$, saturates and is responsible for a nontrivial spatial structure.

Taking into account dilution, we find for $\gamma<1 / 2$ the correlation

$$
\begin{aligned}
& \left\langle\rho(\theta, \phi, t) \rho\left(\theta^{\prime}, \phi^{\prime}, s\right)\right\rangle \\
& =\frac{\min (t, s)[\max (t, s)]^{-2 \gamma}}{4 \pi F^{2}(2 \gamma+1)}+\sum_{l=1}^{\infty} \sum_{m=-l}^{l} \frac{(-1)^{m}}{2 K\left(l+l^{2}\right)} \\
& \quad \times \exp \left[-\frac{K\left(l+l^{2}\right)}{F^{2}(1-2 \gamma)}\left|t^{1-2 \gamma}-s^{1-2 \gamma}\right|\right] Y_{-m}^{l}(\theta, \phi) Y_{m}^{l}\left(\theta^{\prime}, \phi^{\prime}\right),
\end{aligned}
$$

and for $\gamma=1 / 2$,

$$
\begin{aligned}
& \left\langle\rho(\theta, \phi, t) \rho\left(\theta^{\prime}, \phi^{\prime}, s\right)\right\rangle \\
& =\sum_{l=0}^{\infty} \sum_{m=-l}^{l} \frac{(-1)^{m}}{2 F^{2}+2 K\left(l^{2}+l\right)}\left[\frac{\min (s, t)}{\max (s, t)}\right]^{1+K\left(l^{2}+l\right) / F^{2}} \\
& \quad \times Y_{-m}^{l}(\theta, \phi) Y_{m}^{l}\left(\theta^{\prime}, \phi^{\prime}\right)
\end{aligned}
$$


In the two-dimensional situation we see that dilution also has a measurable effect, which is more pronounced in the critical $\gamma=1 / 2$ case. For this value all the modes in the correlation saturate and contribute to create a stationary spatial structure, as in the one-dimensional setting. It is difficult to establish more comparisons among both dimensionalities, as the infinite sums that were explicit in $d=1$ become much more involved in $d=2$, due to the double series containing the spherical harmonics. We nevertheless conjecture that the modification of the scaling properties due to the effect of dilution in two dimensions is similar to the one explicitly observed in one dimension.

As in the preceding section we may wonder what would be the simplest realizations of these processes in the form of discrete systems. Due to the generality of this question, we are not in a position to yield a complete answer to it at this moment. We can, however, point to a direction which seems promising. Several discrete models are known to be in the theoretical university classes described by stochastic growth equations in the classical situation of planar static domains [1]. These models can be cast on a growing, still planar, domain following the technique employed in [25]. This would be a first step in the search for suitable discrete radial models. We note the agreement of the computational results in [25] with our theoretical predictions.

\section{INSTABILITIES}

In this section we analyze the effect that instabilities have on the two-point space-time correlation functions calculated for the processes in Sec. III. A spherical EW equation derived from the geometric principle of surface minimization was introduced in [16]. The corresponding equation for the radius $r(\theta, \phi, t)$ reads

$$
\begin{aligned}
\partial_{t} r= & K\left[\frac{\partial_{\theta} r}{r^{2} \tan (\theta)}+\frac{\partial_{\theta}^{2} r}{r^{2}}+\frac{\partial_{\phi}^{2} r}{r^{2} \sin ^{2}(\theta)}-\frac{2}{r}\right]+F \gamma t^{\gamma-1} \\
& +\frac{1}{r \sqrt{\sin (\theta)}} \xi(\theta, \phi, t)
\end{aligned}
$$

Performing the small noise expansion $r(\theta, \phi, t)=F t+$ $\rho(\theta, \phi, t)$, we find a linear equation which differs from Eq. (46) in that it has a destabilizing term coming from the fourth term in the drift of Eq. (52) (see [16]). In this reference one can see that in the absence of dilution, the $l=0$ mode is unstable and the $l=1$ modes are marginal, while the rest of the modes are stable. The effect of this sort of geometrically originated instability on the mean value of the stochastic perturbation and alternative geometric variational approaches that avoid it can be seen in [16]; herein we concentrate on its effect on correlations. Its effect on mean values can be easily deduced from them.

In the long-time limit and provided $\gamma<1 / 2$, the perturbation is a Gaussian process whose correlation is given by

$$
\begin{aligned}
\left\langle\rho(\theta, \phi, t) \rho\left(\theta^{\prime}, \phi^{\prime}, s\right)\right\rangle= & \frac{1}{16 \pi K} \exp \left[\frac{2 K\left(t^{1-2 \gamma}+s^{1-2 \gamma}\right)}{F^{2}(1-2 \gamma)}\right]+\frac{3[\min (t, s)]^{1-2 \gamma}}{4 \pi F^{2}(1-2 \gamma)}\left[\cos (\theta) \cos \left(\theta^{\prime}\right)+\cos \left(\phi-\phi^{\prime}\right) \sin (\theta) \sin \left(\theta^{\prime}\right)\right] \\
& +\sum_{l=2}^{\infty} \sum_{m=-l}^{l} \frac{(-1)^{m}}{2 K\left(l^{2}+l-2\right)} \exp \left[-\frac{K\left(l^{2}+l-2\right)}{F^{2}(1-2 \gamma)}\left|t^{1-2 \gamma}-s^{1-2 \gamma}\right|\right] Y_{-m}^{l}(\theta, \phi) Y_{m}^{l}\left(\theta^{\prime}, \phi^{\prime}\right) .
\end{aligned}
$$

If $\gamma=1 / 2$, the correlation shifts to

$$
\begin{aligned}
\left\langle\rho(\theta, \phi, t) \rho\left(\theta^{\prime}, \phi^{\prime}, s\right)\right\rangle= & \frac{\left(s t / t_{0}^{2}\right)^{2 K / F^{2}}}{16 \pi K}+\frac{3 \ln [\min (s, t)]}{4 \pi F^{2}}\left[\cos (\theta) \cos \left(\theta^{\prime}\right)+\cos \left(\phi-\phi^{\prime}\right) \sin (\theta) \sin \left(\theta^{\prime}\right)\right] \\
& +\sum_{l=2}^{\infty} \sum_{m=-l}^{l} \frac{(-1)^{m}}{2 K\left(l^{2}+l-2\right)}\left[\frac{\min (s, t)}{\max (s, t)}\right]^{K\left(l^{2}+l-2\right) / F^{2}} Y_{-m}^{l}(\theta, \phi) Y_{m}^{l}\left(\theta^{\prime}, \phi^{\prime}\right) .
\end{aligned}
$$

In these cases the modes characterized by $l=0$ and $l=1$ do not saturate, and the rest of the modes $l>1$ saturate and create a nontrivial spatial structure. When $\gamma<1 / 2$ the $l=1$ modes grow in time as a power law with the exponent $1-2 \gamma$, while the $l=0$ mode grows exponentially fast. When $\gamma=1 / 2$ the $l=1$ modes grow logarithmically and the $l=0$ mode grows as a power law with the nonuniversal exponent $4 K / F^{2}$.

When we consider the effect of dilution, and for $\gamma<1 / 2$, we find the correlation

$$
\begin{aligned}
\left\langle\rho(\theta, \phi, t) \rho\left(\theta^{\prime}, \phi^{\prime}, s\right)\right\rangle= & \frac{1}{16 \pi K} \exp \left[\frac{2 K\left(t^{1-2 \gamma}+s^{1-2 \gamma}\right)}{F^{2}(1-2 \gamma)}\right]+\frac{3 \min (t, s)[\max (t, s)]^{-2 \gamma}}{4 \pi F^{2}(2 \gamma+1)}\left[\cos (\theta) \cos \left(\theta^{\prime}\right)+\cos \left(\phi-\phi^{\prime}\right) \sin (\theta) \sin \left(\theta^{\prime}\right)\right] \\
& +\sum_{l=2}^{\infty} \sum_{m=-l}^{l} \frac{(-1)^{m}}{2 K\left(l^{2}+l-2\right)} \exp \left[-\frac{K\left(l^{2}+l-2\right)}{F^{2}(1-2 \gamma)}\left|t^{1-2 \gamma}-s^{1-2 \gamma}\right|\right] Y_{-m}^{l}(\theta, \phi) Y_{m}^{l}\left(\theta^{\prime}, \phi^{\prime}\right) .
\end{aligned}
$$

For $\gamma=1 / 2$ the correlation reads

$$
\left\langle\rho(\theta, \phi, t) \rho\left(\theta^{\prime}, \phi^{\prime}, s\right)\right\rangle=\frac{1}{4 \pi}\left\langle\rho_{0}^{0}(t) \rho_{0}^{0}(s)\right\rangle+\sum_{l=1}^{\infty} \sum_{m=-l}^{l} \frac{(-1)^{m}}{2 F^{2}+2 K\left(l^{2}+l-2\right)}\left[\frac{\min (s, t)}{\max (s, t)}\right]^{1+K\left(l^{2}+l-2\right) / F^{2}} Y_{-m}^{l}(\theta, \phi) Y_{m}^{l}\left(\theta^{\prime}, \phi^{\prime}\right),
$$


where

$$
\left\langle\rho_{0}^{0}(t) \rho_{0}^{0}(s)\right\rangle=\left\{\begin{array}{lll}
\left(2 F^{2}-4 K\right)^{-1}(\min \{s, t\} / \max \{s, t\})^{1-2 K / F^{2}} & \text { if } & F^{2}>2 K, \\
\ln (\min \{s, t\}) / F^{2} & \text { if } & F^{2}=2 K, \\
\left(4 K-2 F^{2}\right)^{-1}\left(t s / t_{0}^{2}\right)^{2 K / F^{2}-1} & \text { if } & F^{2}<2 K,
\end{array}\right.
$$

where $t_{0}$ is the absolute origin of time.

Contrary to what happens in the stable case, Eq. (46), in the unstable case with no dilution, Eq. (52), the $l=0$ mode is unstable, showing exponential growth, and the $l=1$ modes show an algebraic increase with the universal exponent $1-$ $2 \gamma$, provided $\gamma<1 / 2$; the rest of the modes are stable. The marginal value of the growth index $\gamma=1 / 2$ translates into a power-law increase in the $l=0$ mode with a nonuniversal exponent, while the $l=1$ modes grow logarithmically; the rest of the modes are again stable. It is clear that dilution has a stabilizing effect. Indeed, for $\gamma<1 / 2$ the $l=0$ mode is unchanged, but the $l=1$ modes, which still grow in time, experience a lost of memory effects. In the critical $\gamma=1 / 2$ situation the dilution effects are stronger. The $l=1$ modes, which formerly grew logarithmically, now become stable; the $l=0$ mode, which formerly showed algebraic growth, now show (nonuniversal) algebraic or logarithmic growth, or even saturation, depending on the relation among the values of the parameters of the spherical EW equation. In any case, even that of algebraic growth, this growth is always slower than in the no-dilution situation. Stable modes saturate, contributing to the creation of a nontrivial spatial structure in the whole range $\gamma \leqslant 1 / 2$.

In summary, the effect of dilution is weakly stabilizing in the subcritical case, while stronger and more identifiable in criticality. Of course, the supercritical situation is characterized by an effective random deposition behavior on the large spatial scale.

\section{INTRINSICALLY SPHERICAL GROWTH AND RAPID ROUGHENING}

In this section we show for the first time how rapid roughening naturally appears in the radial growth setting. To this end, it is necessary to clarify the role of the diffusivity index $\zeta$. We have defined it as the order of the fractional differential operator taking mass diffusion into account, and so far we have referred to it as the key element triggering decorrelation. This has been an abuse of language because we have assumed that the negative power of the radius (or its mean field analog $F t^{\gamma}$; what really matters is the resulting power of the temporal variable) preceding this differential operator was exactly $\zeta$. This would not be the case if the diffusion constant were time or radius dependent, but also in some other cases, as the "intrinsically spherical" (IS) equation derived from geometric variational principles in [16]. This equation was obtained as a gradient flow pursuing the minimization of the interface mean curvature and then linearizing with respect to the different derivatives of the radius as given by the small gradient assumption [16]. It is termed IS because it has no planar counterpart, as the nonlinearity becomes fundamental in any attempt to derive such a gradient flow in the Cartesian framework [26,27]. Note the similarity of this to other equations classical in this context, as the EW equation is a gradient flow which minimizes the surface area and the Mullins-Herring equation minimizes the interface square mean curvature [16]; the IS equation, as mentioned, minimizes the interface mean curvature. It reads [16]

$$
\begin{aligned}
\partial_{t} r= & K\left[\frac{\partial_{\theta}^{2} r}{r^{3}}+\frac{\partial_{\phi}^{2} r}{r^{3} \sin ^{2}(\theta)}+\frac{\partial_{\theta} r}{r^{3} \tan (\theta)}-\frac{1}{r^{2}}\right] \\
& +F \gamma t^{\gamma-1}+\frac{1}{r \sqrt{\sin (\theta)}} \xi(\theta, \phi, t),
\end{aligned}
$$

and so $\zeta=2$ in this case; however, one finds a factor $r^{-3}$ in front of the diffusive differential operator, instead of the $r^{-2}$ factor characteristic of the EW equation. This difference has a number of measurable consequences, as we show in the following. The equation for the stochastic perturbation reads, in this case,

$$
\frac{d \rho_{m}^{l}}{d t}=\frac{K}{F^{3} t^{3 \gamma}}[2-l(l+1)] \rho_{m}^{l}-\frac{2 \gamma}{t} \rho_{m}^{l}+\frac{1}{F t^{\gamma}} \eta_{m}^{l}(t),
$$

which reveals that the critical value of the growth index $\gamma=$ $1 / 3$; faster growth leads to decorrelation. This is the first, but not the only, difference with respect to the EW equation. To find out more we first put things in a broader context.

A more general equation for radial growth, after introducing dilution, is

$$
\partial_{t} r=-\frac{K}{r^{\delta}}|\nabla|^{\zeta} r-\frac{\gamma d}{t} r+F \gamma t^{\gamma-1}+\frac{\sqrt{\epsilon}}{\sqrt{r^{d} J(\vec{\theta})}} \eta(\vec{\theta}, t),
$$

which defines the damping index $\delta$, differing from the diffusivity index $\zeta$ in general; note that Eq. (60) has left aside the instability properties of the IS equation, which are analogous to those of the EW equation and would add nothing to the discussion in Sec. IV. For simplicity, we focus on values of the damping index fulfilling $\delta \geqslant \zeta$. This equation can be treated perturbatively for small $\epsilon$ following the procedure in Sec. IV and by introducing the hyperspherical harmonics $Y_{l}^{\vec{m}}(\vec{\theta})$, which obey the eigenvalue equation [28]

$$
\nabla^{2} Y_{l}^{\vec{m}}(\vec{\theta})=-l(l+d-1) Y_{l}^{\vec{m}}(\vec{\theta})
$$

where the vector $\vec{m}$ represents the set of $(d-1)$ indices. The fractional operator acts on the hyperspherical harmonics in the following fashion:

$$
|\nabla|^{\zeta} Y_{l}^{\vec{m}}(\vec{\theta})=[l(l+d-1)]^{\zeta / 2} Y_{l}^{\vec{m}}(\vec{\theta}) .
$$

The hyperspherical noise is Gaussian and has zero mean, and its correlation is given by

$$
\left\langle\eta(\vec{\theta}, t) \eta\left(\vec{\theta}^{\prime}, t^{\prime}\right)\right\rangle=\delta\left(\vec{\theta}-\vec{\theta}^{\prime}\right) \delta\left(t-t^{\prime}\right) .
$$


It can be expanded in terms of hyperspherical harmonics,

$$
\frac{\eta(\vec{\theta}, t)}{\sqrt{J(\vec{\theta})}}=\sum_{l, \vec{m}} \eta_{l}^{\vec{m}}(t) Y_{l}^{\vec{m}}(\vec{\theta}),
$$

and the amplitudes are given by

$$
\eta_{l}^{\vec{m}}(t)=\int \eta(\vec{\theta}, t) \bar{Y}_{l}^{\vec{m}}(\vec{\theta}) \sqrt{J(\vec{\theta})} d \vec{\theta},
$$

and so they are zero-mean Gaussian noises whose correlation is given by

$$
\left\langle\eta_{l}^{\vec{m}}(t) \bar{\eta}_{l^{\prime}}^{\vec{m}^{\prime}}\left(t^{\prime}\right)\right\rangle=\delta\left(t-t^{\prime}\right) \delta_{l, l^{\prime}} \delta_{\vec{m}, \vec{m}^{\prime}},
$$

where the overbar denotes complex conjugation. Note that the amplitudes are in general complex valued. They obey the linear equation

$$
\begin{aligned}
\frac{d \rho_{l}^{\vec{m}}}{d t}= & -\frac{K}{F^{\delta} t^{\delta \gamma}}[l(l+d-1)]^{\zeta / 2} \rho_{l}^{\vec{m}}-\frac{\gamma d}{t} \rho_{l}^{\vec{m}} \\
& +\frac{1}{F^{d / 2} t^{\gamma d / 2}} \eta_{l}^{\vec{m}}(t) .
\end{aligned}
$$

From this equation it is clear that the critical value of the growth index is $\gamma=1 / \delta$, and faster growth leads to decorrelation.

It is convenient to move to a growing hypercubic geometry as in [17] in order to calculate different quantities,

$$
\begin{aligned}
\partial_{t} h= & -D\left(\frac{t_{0}}{t}\right)^{\delta \gamma}|\nabla|^{\zeta} h-\frac{d \gamma}{t} h+\gamma F t^{\gamma-1} \\
& +\left(\frac{t_{0}}{t}\right)^{d \gamma / 2} \xi(x, t),
\end{aligned}
$$

since this change simplifies calculations without modifying the leading results. Our goal is finding the growth and autocorrelation exponents, as the latter is a good quantity for measuring decorrelation [17]. In order to calculate the temporal correlations we need to consider the short-time limit, where the growth exponent $\beta$ becomes apparent. The propagator of Eq. (68) is

$$
G_{n}(t)=\left(\frac{t}{t_{0}}\right)^{-d \gamma} \exp \left[-\frac{n^{\zeta} \pi^{\zeta} D}{L_{0}^{\zeta}} \frac{t_{0}^{\gamma \delta} t^{1-\gamma \delta}-t_{0}}{1-\gamma \delta}\right],
$$

which yields the following complete solution when the initial condition vanishes:

$$
h_{n}(t)=G_{n}(t) \int_{t_{0}}^{t} G_{n}^{-1}(\tau)\left(\frac{t_{0}}{\tau}\right)^{d \gamma / 2} \xi_{n}(\tau) d \tau
$$

The one-point two-times correlation function then reads

$$
\left\langle h_{n}(t) h_{n}\left(t^{\prime}\right)\right\rangle \sim G_{n}(t) G_{n}\left(t^{\prime}\right) \int_{t_{0}}^{\min \left(t, t^{\prime}\right)} G_{n}^{-2}(\tau)\left(\frac{t_{0}}{\tau}\right)^{d \gamma} d \tau,
$$

and after inverting Fourier we arrive at the real-space expression

$$
\left\langle h(x, t) h\left(x, t^{\prime}\right)\right\rangle=\sum_{n=0}^{\infty}\left\langle h_{n}(t) h_{n}\left(t^{\prime}\right)\right\rangle \cos ^{2}\left(\frac{n \pi x}{L_{0}}\right),
$$

where we have assumed no flux boundary conditions as in [17], although the values of both the growth and the autocorrelation exponents do not depend on the choice of boundary conditions. The propagator $G_{n}(t)$ suggests the scaling variable $v_{n} \sim$ $n t^{(1-\gamma \delta) / \zeta}$ in Fourier space, which corresponds to the real-space scaling variable $u \sim x t^{(-1+\gamma \delta) / \zeta}$, as can be read directly from Eq. (72). This suggests the definition of the effective dynamical exponent $z_{\mathrm{eff}}=\zeta /(1-\gamma \delta)$. If we express the correlation, Eq. (71), for $t=t^{\prime}$ in terms of the scaling variable $v_{n}$ [and we refer to it as $C\left(v_{n}\right)$ multiplied by a suitable power of $t$ ] and we introduce the "differential" $1 \equiv \Delta n \sim t^{(-1+\gamma \delta) / \zeta} \Delta v$, we can cast the last expression in the integral form,

$$
\begin{aligned}
\left\langle h(x, t)^{2}\right\rangle-\langle h(x, t)\rangle^{2}= & t^{1-d / \zeta+\gamma d(\delta / \zeta-1)} \int_{v_{1}}^{\infty} C\left(v_{n}\right) \\
& \times \cos ^{2}\left(\frac{v_{n} \pi u}{L_{0}}\right) d v_{n},
\end{aligned}
$$

where the series converges as a Riemann sum to the above integral when

$$
D t \ll\left(L_{0}^{\zeta}+D t_{0}\right) \frac{t^{\delta \gamma}}{t_{0}^{\delta \gamma}}
$$

or, equivalently, $t \ll t_{c} \sim L_{0}^{z_{\text {eff }}}$, with $t_{c}$ being the time it takes the correlations to reach the substrate boundaries, assuming that the initial substrate size is very large. If $\gamma<1 / \delta$, the whole substrate becomes correlated, yielding a finite $t_{c}$; for $\gamma>1 / \delta$ the convergence of the Riemann sum to the integral is assured for all times, corresponding to the physical fact that the substrate never becomes correlated. In front of the integral we find a power of the temporal variable compatible with the growth exponent

$$
\beta=\frac{1}{2}-\frac{d}{2 \zeta}+\frac{\gamma d}{2}\left(\frac{\delta}{\zeta}-1\right),
$$

and the integral can be shown to be absolutely convergent as the integrand decays more rapidly than exponentially for large values of the scaling variable $v_{n}$.

We are now in position to calculate the temporal autocorrelation

$$
A\left(t, t^{\prime}\right) \equiv \frac{\left\langle h(x, t) h\left(x, t^{\prime}\right)\right\rangle_{0}}{\left\langle h(x, t)^{2}\right\rangle_{0}^{1 / 2}\left\langle h\left(x, t^{\prime}\right)^{2}\right\rangle_{0}^{1 / 2}} \sim\left(\frac{\min \left\{t, t^{\prime}\right\}}{\max \left\{t, t^{\prime}\right\}}\right)^{\lambda},
$$

where $\lambda$ is the autocorrelation exponent and $\langle\cdot\rangle_{0}$ denotes the average with the zeroth mode contribution suppressed, as in (73). The remaining ingredient is the correlation $\left\langle h(x, t) h\left(x, t^{\prime}\right)\right\rangle_{0}$. Going back to Eq. (72) we see that the Fourier space scaling variable now reads

$$
v_{n}=\left[\frac{t^{1-\gamma \delta}+\left(t^{\prime}\right)^{1-\gamma \delta}-2 \tau^{1-\gamma \delta}}{1-\gamma \delta}\right]^{1 / \zeta} n .
$$

If $\gamma<1 / \delta$, the term $\max \left\{t, t^{\prime}\right\}^{1-\gamma \delta}$ is dominant and the factor in front of the convergent Riemann sum reads

$$
\max \left\{t, t^{\prime}\right\}^{(\delta / \zeta-1) \gamma d-d / \zeta} \min \left\{t, t^{\prime}\right\},
$$

after the time integration has been performed and in the limit $\max \left\{t, t^{\prime}\right\} \gg \min \left\{t, t^{\prime}\right\}$. In this same limit, but when $\gamma>1 / \delta$, the term $\min \left\{t, t^{\prime}\right\}^{1-\gamma \delta}$ becomes dominant and the prefactor reads

$$
\max \left\{t, t^{\prime}\right\}^{-d \gamma} \min \left\{t, t^{\prime}\right\}^{1-d / \zeta+d \gamma \delta / \zeta}
$$


The resulting temporal correlation adopts the form indicated on the right-hand side of (76), where

$$
\lambda= \begin{cases}\beta+d / \zeta+\gamma d(1-\delta / \zeta) & \text { if } \quad \gamma<1 / \delta \\ \beta+\gamma d & \text { if } \quad \gamma>1 / \delta\end{cases}
$$

or, alternatively,

$$
\lambda=\beta+\frac{d}{z_{\lambda}},
$$

where the $\lambda$-dynamical exponent is defined as

$$
z_{\lambda}=\left\{\begin{array}{lll}
\frac{\zeta}{1+\gamma(\zeta-\delta)} & \text { if } & \gamma<1 / \delta \\
1 / \gamma & \text { if } & \gamma>1 / \delta
\end{array}\right.
$$

If we disregarded the effect of dilution, we would again find Eq. (81), but this time

$$
z_{\lambda}=\left\{\begin{array}{lll}
\frac{\zeta}{1-\gamma \delta}=z_{\text {eff }} & \text { if } & \gamma<1 / \delta \\
\infty & \text { if } & \gamma>1 / \delta
\end{array}\right.
$$

To further clarify the dynamics we now calculate the scaling form that the two-points correlation function adopts for short spatial scales $\left|x-x^{\prime}\right| \ll t^{(1-\delta \gamma) / \zeta}$ in the decorrelated regime. As dilution does not act on such a microscopic scale, the following results are independent of whether or not we contemplate dilution. In this case one has

$$
\begin{aligned}
\left\langle h(x, t) h\left(x^{\prime}, t\right)\right\rangle= & \sum_{n_{1}, \ldots, n_{d}}\left\langle h_{n}^{2}(t)\right\rangle \cos \left(\frac{n_{1} \pi x_{1}}{L_{0}}\right) \cos \left(\frac{n_{1} \pi x_{1}^{\prime}}{L_{0}}\right) \\
& \cdots \cos \left(\frac{n_{d} \pi x_{d}}{L_{0}}\right) \cos \left(\frac{n_{d} \pi x_{d}^{\prime}}{L_{0}}\right),
\end{aligned}
$$

where $x=\left(x_{1}, \ldots, x_{d}\right)$ and $n=\left(n_{1}, \ldots, n_{d}\right)$, and we assume the rough interface inequality $\zeta>d$ in order to assure the absolute convergence of this expression. By introducing the scaling variables $v_{i}=n_{i} t^{(1-\delta \gamma) / \zeta}$ and $u_{i}=x_{i} t^{(\gamma \delta-1) / \zeta}$ for $i=$ $1, \ldots, d$ and assuming statistical isotropy and homogeneity of the scaling form, we find

$$
\begin{aligned}
& \left\langle h(x, t) h\left(x^{\prime}, t\right)\right\rangle-\langle h(x, t)\rangle^{2} \\
& \quad=\left|x-x^{\prime}\right|^{\zeta-d} t^{\gamma(\delta-d)} \mathcal{F}\left[\left|x-x^{\prime}\right| t^{(\delta \gamma-1) / \zeta}\right]
\end{aligned}
$$

or, in Lagrangian coordinates $\left|y-y^{\prime}\right|=\left|x-x^{\prime}\right| t^{\gamma}$,

$$
\begin{aligned}
& \left\langle h(y, t) h\left(y^{\prime}, t\right)\right\rangle-\langle h(y, t)\rangle^{2} \\
& \quad=\left|y-y^{\prime}\right|^{\zeta-d} t^{\gamma(\delta-\zeta)} \mathcal{F}\left[\frac{\left|y-y^{\prime}\right|}{t^{\{1+\gamma(\zeta-\delta)\} / \zeta}}\right] .
\end{aligned}
$$

We see that this form is statistically self-affine with respect to the rescaling $y \rightarrow b y, t \rightarrow b^{z} t$, and $h \rightarrow b^{\alpha} h$, where the critical exponents are

$\alpha=\frac{\zeta-d}{2}+\frac{\zeta}{1+\gamma(\zeta-\delta)} \frac{(\delta-\zeta) \gamma}{2}, \quad z=\frac{\zeta}{1+\gamma(\zeta-\delta)}$.

Note that the scaling relation $\alpha=\beta z$ holds, where the growth exponent $\beta$ was calculated in Eq. (75). The macroscopic decorrelation, which is observed for length scales of the order of the system size $\left|x-x^{\prime}\right| \approx L_{0}$, is controlled by the effective dynamical exponent $z_{\text {eff }}$. When $\delta>\zeta$, decorrelation might happen at microscopic length scales $\left|x-x^{\prime}\right| \ll t^{(1-\delta \gamma) / \zeta}$ as well. Microscopic decorrelation happens in the limit $\delta \rightarrow \zeta+1 / \gamma$. For $\delta<\zeta+1 / \gamma$ the interface is microscopically correlated and the critical exponents take on their finite values given in Eq. (87). For $\delta \geqslant \zeta+1 / \gamma$ the interface is microscopically uncorrelated and the critical exponents diverge, $\alpha=z=\infty$, while the growth exponent is still finite and given by Eq. (75) (so one could say that the scaling relation $\alpha=\beta z$ still holds in some sense in the microscopic uncorrelated limit). With respect to the growth exponent we can say that $\beta<1 / 2$ when $\delta<\gamma^{-1}+\zeta, \beta \rightarrow 1 / 2$ when $\delta \rightarrow \gamma^{-1}+\zeta$, and $\beta>1 / 2$ when $\delta>\gamma^{-1}+\zeta$, so rapid roughening is a consequence of microscopic decorrelation. And now, by applying the developed theory to the IS equation, for which $d=2, \zeta=2$, and $\delta=3$, and assuming, as in [16], that $\gamma=1$, we find that it is exactly positioned at the threshold of microscopic decorrelation; that is, its critical exponents are $\alpha=z=\infty$ and $\beta=1 / 2$.

Note that the effective dynamical exponent $z_{\text {eff }}=\zeta /(1-$ $\gamma \delta$ ) states the speed at which both correlation and decorrelation occur. The transition from correlation to decorrelation is triggered by the comparison among the indexes $\gamma$ and $\delta$. The derivation order $\zeta$ controls the speed at which both processes happen: a larger $\zeta$ implies slower correlation and decorrelation processes. Note also that rapid roughening might appear in exactly the same way in planar processes, just by allowing field or time dependence on the diffusion constant. This is actually the case in some planar situations [29], and we have also shown that it appears naturally in the radial case, where such a dependence is a straightforward consequence of the lost of translation invariance, due to the existence of an absolute origin of space, characterized by a zero radius (and which, in turn, implies the existence of an absolute origin of time in the small noise approximation, as we have already seen). Such a naturalness can be seen in the derivation of the IS equation in [16], where it was found as a consequence of a simple variational principle.

\section{SCALE DEPENDENT FRACTALITY}

We devote this section to showing, for the first time, that rapidly growing radial interfaces develop scale-dependent fractality. This expression denotes a behavior characterized by a scale-dependent fractal dimension taking place in a finite system and for long times. It is different from the concept of multifractality, which in this topic is usually associated with a nonlinear relation among the exponents characterizing the higher order height difference correlations [1].

In the classical case of static planar interfaces the fractal dimension is computed from the height difference correlation function,

$$
\left\langle\left[h(x, t)-h\left(x^{\prime}, t\right)\right]^{2}\right\rangle^{1 / 2} \sim\left|x-x^{\prime}\right|^{H},
$$

in the long-time limit, i.e., after saturation have been achieved, where the Hurst exponent $H=(\zeta-d) / 2$ for linear growth equations and the right-hand side is time independent. The interface fractional dimension is calculated using the box counting method and is given by $d_{f}=1+d-H$. The general 
linear equation for stochastic growth in a growing domain was found in Sec. V to be

$$
\begin{aligned}
\partial_{t} h= & -D\left(\frac{t_{0}}{t}\right)^{\delta \gamma}|\nabla|^{\zeta} h-\frac{d \gamma}{t} h \\
& +\gamma F t^{\gamma-1}+\left(\frac{t_{0}}{t}\right)^{d \gamma / 2} \xi(x, t),
\end{aligned}
$$

for which we assume $\zeta \leqslant \delta<\zeta+\gamma^{-1}$. Its Fourier transformed version, for $n \geqslant 1$, is

$$
\frac{d h_{n}}{d t}=-D\left(\frac{t_{0}}{t}\right)^{\delta \gamma} \frac{\pi^{\zeta}|n|^{\zeta}}{L_{0}^{\zeta}} h_{n}-\frac{d \gamma}{t} h_{n}+\left(\frac{t_{0}}{t}\right)^{d \gamma / 2} \xi_{n}(t) .
$$

For slow growth $\gamma<1 / \delta$ diffusion dominates over dilution and one finds an expression compatible with that in the planar case,

$$
\left\langle\left[h(x, t)-h\left(x^{\prime}, t\right)\right]^{2}\right\rangle^{1 / 2} \sim t^{\gamma(\delta-d) / 2}\left|x-x^{\prime}\right|^{(\zeta-d) / 2},
$$

and so the Hurst exponent and interface fractal dimension are the same as in the planar case for a fixed time. In the case of fast growth $\gamma>1 / \delta$, for small spatial scales $\left|x-x^{\prime}\right| \ll t^{(1-\delta \gamma) / \zeta}$, we again recover this result, while for large spatial scales $\left|x-x^{\prime}\right| \gg t^{(1-\delta \gamma) / \zeta}$ we find

$$
\left\langle\left[h(x, t)-h\left(x^{\prime}, t\right)\right]^{2}\right\rangle^{1 / 2} \sim t^{\beta},
$$

and so, for a fixed time, $H=0$ and $d_{f}=d+1$. This means that the interface becomes highly irregular and so dense that it fills the $(d+1)$-dimensional space. In this way decorrelation marks the onset of scale-dependent fractality, as specified by a scale-dependent Hurst exponent, whose asymptotic values ar

$$
H\left(\left|x-x^{\prime}\right|, t\right)=\left\{\begin{array}{lll}
(\zeta-d) / 2 & \text { if } & \left|x-x^{\prime}\right| \ll t^{(1-\delta \gamma) / \zeta}, \\
0 & \text { if } & \left|x-x^{\prime}\right| \gg t^{(1-\delta \gamma) / \zeta},
\end{array}\right.
$$

and the corresponding asymptotic values of the scaledependent fractal dimension

$$
\begin{aligned}
& d_{f}\left(\left|x-x^{\prime}\right|, t\right) \\
& \quad=\left\{\begin{array}{lll}
1+(3 d-\zeta) / 2 & \text { if } & \left|x-x^{\prime}\right| \ll t^{(1-\delta \gamma) / \zeta}, \\
d+1 & \text { if } & \left|x-x^{\prime}\right| \gg t^{(1-\delta \gamma) / \zeta} .
\end{array}\right.
\end{aligned}
$$

Note that these results imply dynamic scale-dependent fractality, as the scale separating the two regimes depends on time, $\left|x-x^{\prime}\right| \sim t^{(1-\delta \gamma) / \zeta}$; also, the rough interface inequality $\zeta>d$ implies the strict inequality $1+(3 d-\zeta) / 2<d+1$. This asymptotic behavior strongly suggests the self-similar form of both the Hurst exponent and the fractal dimension

$$
H=H\left(\frac{\left|x-x^{\prime}\right|}{t^{(1-\delta \gamma) / \zeta}}\right) \quad \text { and } \quad d_{f}=d_{f}\left(\frac{\left|x-x^{\prime}\right|}{t^{(1-\delta \gamma) / \zeta}}\right) .
$$

According to this, the fractal dimension would be a dynamic fractal itself, invariant to the transformation $x \rightarrow b x$, $t \rightarrow b^{z_{f}} t$, and $d_{f} \rightarrow b^{\alpha_{f}} d_{f}$, for $z_{f}=\zeta /(1-\delta \gamma)=z_{\text {eff }}$ and $\alpha_{f}=0$. Note that all these results concerning scale-dependent fractality are independent of whether or not we contemplate dilution (because the height difference correlation function depends on strictly local quantities [17]), and so we could, in this particular calculation, substitute Eqs. (89) and (90) with their dilution-free counterparts and still get the same results. Note also that at the very beginning of this section we have assumed the inequality $\zeta \leqslant \delta<\zeta+\gamma^{-1}$, which implies that for rapid growth the interface is macroscopically but not microscopically uncorrelated. If $\delta \geqslant \zeta+\gamma^{-1}$, then the interface is microscopically uncorrelated and the fractal dimension becomes $d_{f}=d+1$ independently of the scale from which we regard it; i.e., scale-dependent fractality is a genuine effect of macroscopic decorrelation, which disappears for strong damping causing microscopic decorrelation.

Note that scale-dependent fractality does not appear in nongrowing domain systems, as for long times saturation is achieved and the fractal dimension becomes constant (assuming that no multifractality is present). Although the behavior of the height difference correlation function we found here is similar to that of the one present in classical unbounded systems, results concerning the fractal dimension cannot be immediately extrapolated. The fractal dimension can be computed in a bounded growing domain, e.g., using the box counting method as we have done herein, by employing as the reference length $L(t)$, the linear time-dependent size of the system. Of course, in an unbounded static domain there is not such a reference length.

\section{THE KARDAR-PARISI-ZHANG EQUATION}

One of the most important nonlinear models in the field of surface growth is the Kardar-Parisi-Zhang (KPZ) equation [30]:

$$
\partial_{t} h=v \nabla^{2} h+\lambda(\nabla h)^{2}+\xi(x, t)
$$

It is related to the biologically motivated Eden model, as this model, at least in a planar geometry, was numerically found to belong to the KPZ universality class [1]. As we see, understanding the KPZ equation in a growing domain may shed some light on some of the properties of the classical version of this model.

The KPZ equation in a growing domain reads

$$
\begin{aligned}
\partial_{t} h= & v\left(\frac{t_{0}}{t}\right)^{2 \gamma} \nabla^{2} h+\frac{\lambda}{2}\left(\frac{t_{0}}{t}\right)^{2 \gamma}(\nabla h)^{2}-\frac{d \gamma}{t} h+\gamma F t^{\gamma-1} \\
& +\left(\frac{t_{0}}{t}\right)^{d \gamma / 2} \xi(x, t) .
\end{aligned}
$$

Of course, if we just considered the dilatation $x \rightarrow\left(t / t_{0}\right)^{\gamma} x$, we would find

$$
\begin{aligned}
\partial_{t} h= & v\left(\frac{t_{0}}{t}\right)^{2 \gamma} \nabla^{2} h+\frac{\lambda}{2}\left(\frac{t_{0}}{t}\right)^{2 \gamma}(\nabla h)^{2}+\gamma F t^{\gamma-1} \\
& +\left(\frac{t_{0}}{t}\right)^{d \gamma / 2} \xi(x, t) .
\end{aligned}
$$

As we have shown in Sec. VI, the dilution mechanism fixes the Family-Vicsek scaling in the fast-growth regime. In the radial Eden model case, assuming it belongs to the KPZ universality class, we would have $z=3 / 2$ in $d=1$ and $\gamma=1$. And so, one would naively expect that the resulting interface is uncorrelated and we have to resort to dilution effects in order to fix the Family-Vicsek ansatz and get rid of memory effects. But here comes the paradoxical situation. There are 
two main symmetries associated with the $d$-dimensional KPZ equation: the Hopf-Cole transformation, which maps it onto the noisy diffusion equation [31] and the related directed polymer problem [32,33]; and Galilean invariance, which has traditionally been related to the nonrenormalization of the KPZ vertex at an arbitrary order in the perturbation expansion $[34,35]$. In the case of the no-dilution KPZ equation (98), both symmetries are still present. Indeed, this equation transforms under the Hopf-Cole transformation $u=\exp [\lambda h /(2 v)]$ to

$\partial_{t} u=v\left(\frac{t_{0}}{t}\right)^{2 \gamma} \nabla^{2} u+\frac{\gamma F \lambda}{2 v} t^{\gamma-1} u+\frac{\lambda}{2 v}\left(\frac{t_{0}}{t}\right)^{d \gamma / 2} \xi(x, t) u$,

which is again a noisy diffusion equation, and it can be explicitly solved in the deterministic limit $\epsilon=0$. We find, in this case,

$$
\begin{aligned}
u(x, t)= & \frac{(1-2 \gamma)^{d / 2} \exp \left[F \lambda t^{\gamma} /(2 \nu)\right]}{\left[4 \pi t_{0}^{2 \gamma}\left(t^{1-2 \gamma}-t_{0}^{1-2 \gamma}\right)\right]^{d / 2}} \\
& \times \int_{\mathbb{R}^{d}} \exp \left[-\frac{|x-y|^{2}(1-2 \gamma)}{4 t_{0}^{2 \gamma}\left(t^{1-2 \gamma}-t_{0}^{1-2 \gamma}\right)}\right] u\left(y, t_{0}\right) d y,
\end{aligned}
$$

which corresponds to

$$
\begin{aligned}
& h(x, t) \\
& =\frac{2 \nu}{\lambda} \ln \left\{\frac{(1-2 \gamma)^{d / 2} \exp \left[F \lambda t^{\gamma} /(2 \nu)\right]}{\left[4 \pi t_{0}^{2 \gamma}\left(t^{1-2 \gamma}-t_{0}^{1-2 \gamma}\right)\right]^{d / 2}}\right. \\
& \left.\quad \times \int_{\mathbb{R}^{d}} \exp \left[-\frac{|x-y|^{2}(1-2 \gamma)}{4 t_{0}^{2 \gamma}\left(t^{1-2 \gamma}-t_{0}^{1-2 \gamma}\right)}+\frac{\lambda}{2 \nu} h\left(y, t_{0}\right)\right] d y\right\}
\end{aligned}
$$

for given initial conditions $u\left(x, t_{0}\right)$ and $h\left(x, t_{0}\right)$. Note that we are using the same shorthand notation for differentials and coordinates as in Sec. I. It is clear from regarding this formula that decorrelation at the deterministic level will happen for $\gamma>1 / 2$. It is still necessary to find out if, at the stochastic level, this threshold will be moved to $\gamma>2 / 3$. If we consider the dilution KPZ equation (97), then transforming Hopf-Cole, we would find the nonlinear equation

$$
\begin{aligned}
\partial_{t} u= & v\left(\frac{t_{0}}{t}\right)^{2 \gamma} \nabla^{2} u-\frac{d \gamma}{t} u \ln (u)+\frac{\gamma F \lambda}{2 v} t^{\gamma-1} u \\
& +\frac{\lambda}{2 v}\left(\frac{t_{0}}{t}\right)^{d \gamma / 2} \xi(x, t) u
\end{aligned}
$$

which may be thought of as a time-dependent and spatially distributed version of the Gompertz differential equation [36]. In this case it is not evident how to find an explicit solution at the deterministic level and what its decorrelation threshold would be.

Galilean invariance means that the transformation

$$
x \rightarrow x-\lambda v t, \quad h \rightarrow h+v x, \quad F \rightarrow F-\frac{\lambda}{2} v^{2},
$$

where $v$ is an arbitrary constant vector field, leaves the KPZ equation invariant. In the case of no dilution this transformation can be replaced by

$$
\begin{aligned}
& x \rightarrow x-\frac{\lambda}{1-2 \gamma} v t_{0}^{2 \gamma} t^{1-2 \gamma}, \quad h \rightarrow h+v x, \\
& F \rightarrow F-\frac{\lambda}{2 \gamma} v^{2} t_{0}^{2 \gamma} t^{1-3 \gamma},
\end{aligned}
$$

which leaves invariant Eq. (98). If we consider dilution, then it is not clear how to extend this transformation to leave Eq. (97) invariant. The main difficulty comes from the dilution term, which yields a nonhomogeneous contribution to the dynamics as a response to the rotation $h \rightarrow h+v x$. So in summary, we may talk of a certain sort of Galilean invariance which is obeyed by the no-dilution KPZ dynamics (98), and is lost when dilution is taken into account. If it were found that the dilution equation (97), obeys the traditional KPZ scaling (at least in some suitable limit), then that would mean the possible necessity of readdressing the role that the symmetries of the KPZ equation have in fixing the universality class [37-41].

There is still another fundamental symmetry of the KPZ equation, but this time it manifests itself in just one spatial dimension: the so-called fluctuation-dissipation theorem [1]. It basically says that for long times, when saturation has already being achieved, nonlinearity ceases to be operative and the resulting interface profile would be statistically indistinguishable from that created by the EW equation. For fast domain growth, we know from the linear theory that the interface never becomes correlated, and it operates, in this sense, as if it were effectively in the short-time regime for all times [17]. As a consequence, the fluctuation-dissipation theorem is not expected to play any role in this case. Of course, this result would be independent of whether or not we contemplated dilution.

In more general terms, it is known that the different symmetries of statistical mechanical models influence their scaling properties $[42,43]$. It would be interesting to understand in complete generality the interplay among the symmetries of a physical model in a static domain and the asymmetric presence of dilution when we let this domain grow in time. A possible framework to carry out this project is the instanton approach for the KPZ equation developed in [44] and [45]. Complimentarily, this approach motivates an interesting theoretical problem, which is the development of appropriate techniques to deal with an explicitly time-dependent Martin-Siggia-Rose theory, that is, with a nonautonomous infinite-dimensional Hamiltonian dynamical system.

\section{CENTER-OF-MASS FLUCTUATIONS}

Another property that has been studied in the context of radial growth, particularly in Eden clusters, is the center-ofmass fluctuations. In this section we derive for the first time the properties of the center-of mass fluctuations of the cluster interfaces described by radial stochastic growth equations. It was found numerically that the Eden center of mass fluctuates according to the power law $C_{m} \sim t^{2 / 5}$ in $d=1+1$ [4], while in $d=2+1$ there is a strong decrease in this exponent [46]. This reduced stochastic behavior in higher dimensions was 
predicted in [15] using radial growth equations, and here we examine further the compatibility among the equations and the Eden cluster dynamics. The center-of-mass fluctuations are characteristic not only of radial growth but also of planar situations. Let us recall the classical EW equation,

$$
\partial_{t} h=D \nabla^{2} h+\xi(x, t),
$$

defined in a one-dimensional domain of linear size $L_{0}$ and with no flux boundary conditions. It is straightforward to find that the center of mass $h_{0}(t)=L_{0}^{-1} \int_{0}^{L_{0}} h(x, t) d x$ is a Gaussian stochastic process defined by its two first moments,

$$
\left\langle h_{0}(t)\right\rangle=0, \quad\left\langle h_{0}(t) h_{0}(s)\right\rangle=\frac{\epsilon}{L_{0}} \min (t, s),
$$

and so we have found that the center of mass performs Brownian motion, or equivalently, we would say that its position is given by a Wiener process. Note that the fluctuation amplitude decreases with the linear system size, suggesting that in the case of a growing domain, our current law $C_{m}=$ $\left\langle h_{0}^{2}\right\rangle^{1 / 2} \sim t^{1 / 2}$ will be replaced by a different power law with a smaller exponent. It is easy to see that this result does not hold uniquely for the one-dimensional EW equation; indeed, for any $d$-dimensional growth equation with a conserved growth mechanism, be it linear as the EW or Mullins-Herring equations [1] or nonlinear as the Villain-Lai-Das Sarma equation [47,48] or its Monge-Ampère variation [26], the center of mass performs Brownian motion characterized by the correlators

$$
\left\langle h_{0}(t)\right\rangle=0, \quad\left\langle h_{0}(t) h_{0}(s)\right\rangle=\frac{\epsilon}{L_{0}^{d}} \min (t, s),
$$

as a consequence of the decoupling of the zeroth mode with respect to the surface fluctuations [47]. Note that in the case of nonconserved growth dynamics, this is not the case, as illustrated by the KPZ equation,

$$
\partial_{t} h=v \nabla^{2} h+\lambda(\nabla h)^{2}+\xi(x, t) .
$$

It is easy to see that in this case

$$
\frac{d h_{0}}{d t}=\frac{\lambda}{L^{d}} \int(\nabla h)^{2} d x+\xi_{0}(t) \geqslant \xi_{0}(t),
$$

where $\xi_{0}(t)=L^{-d} \int \xi(x, t) d x$ and the equal sign is attained only for $h=$ constant, an unstable configuration for KPZ dynamics. And so one expects stronger center-of-mass fluctuations in this case. Actually, the short-time center-of-mass fluctuations can be easily calculated for any model which obeys the Family-Vicsek scaling, including the KPZ equation. Indeed, the Family-Vicsek scaling implies the following form of the height-height correlation,

$$
\left\langle h(x, t) h\left(x^{\prime}, t\right)\right\rangle=t^{2 \beta} C\left(\frac{\left|x-x^{\prime}\right|}{t^{1 / z}}\right),
$$

which in the short-time limit reduces to

$$
\left\langle h(x, t) h\left(x^{\prime}, t\right)\right\rangle \sim t^{2 \beta+d / z} \delta\left(x-x^{\prime}\right),
$$

leading to the result

$$
\left\langle h_{0}(t)^{2}\right\rangle \sim L^{-d} t^{2 \beta+d / z} .
$$

And so, within the Family-Vicsek scaling framework, the exponent characterizing the short-time behavior of the centerof-mass fluctuations is $\beta+d /(2 z)$.

As we have seen, the center-of-mass fluctuations are given by the zeroth mode. In the growing domain case it can be shown that the equation controlling the evolution of $h_{0}$ is [17]

$$
\frac{d h_{0}}{d t}=-\frac{d \gamma}{t} h_{0}+\gamma F t^{\gamma-1}+\left(\frac{t_{0}}{t}\right)^{d \gamma / 2} \xi_{0}(t),
$$

in the case where dilution is taken into account. In this case we find, for long times, the center-of-mass fluctuations

$$
C_{m}^{2}=\left\langle h_{0}(t)^{2}\right\rangle-\left\langle h_{0}(t)\right\rangle^{2}=\frac{\epsilon t_{0}^{d \gamma}}{L_{0}^{d}(d \gamma+1)} t^{1-d \gamma},
$$

and so $C_{m} \sim t^{(1-d \gamma) / 2}$. If we did not consider dilution, we would find, in the long-time limit,

$$
C_{m}^{2}=\left\{\begin{array}{lll}
\frac{\epsilon t_{0}^{d \gamma}}{L_{0}^{d}(1-d \gamma)} t^{1-d \gamma} & \text { if } & \gamma<1 / d, \\
\frac{\epsilon t_{0}}{L_{0}^{d}} \ln (t) & \text { if } & \gamma=1 / d, \\
\frac{\epsilon t_{0}}{L_{0}^{d}(d \gamma-1)} & \text { if } & \gamma>1 / d .
\end{array}\right.
$$

If we adapt result (112) to the present setting, we find

$$
\begin{aligned}
& \left\langle h(x, t) h\left(x^{\prime}, t\right)\right\rangle \sim t^{2 \beta+d / z} \delta\left[t^{\gamma}\left(x-x^{\prime}\right)\right] \\
& \quad=t^{2 \beta+d / z-\gamma d} \delta\left(x-x^{\prime}\right) .
\end{aligned}
$$

For linear systems the equality $2 \beta+d / z=1$ holds, and so this last equation agrees with (114) but not with (115). This is a consequence of the violation of the Family-Vicsek scaling in the absence of dilution $[17,19]$. In the case of the $(1+1)-$ dimensional Eden model $d=\gamma=1$, and if it belonged to the KPZ universality class, the center of mass would fluctuate according to the law $C_{m} \sim t^{1 / 6}$. This of course does not agree with the measured behavior $C_{m} \sim t^{2 / 5}$. This exponent could be recovered by introducing an ad hoc instability mechanism, such as considering a growth equation whose zeroth moment obeyed

$$
\frac{d h_{0}}{d t}=D\left(\frac{t_{0}}{t}\right)^{\delta \gamma} h_{0}+\gamma F t^{\gamma-1}+\left(\frac{t_{0}}{t}\right)^{d \gamma / 2} \xi_{0}(t) .
$$

The desired exponent is obtained for $\delta=1$ and $D t_{0}=2 / 5$, however, this result is uniform in the spatial dimension and so cannot predict the $(2+1)$-dimensional behavior [46]. Additionally, this instability mechanism seems to be not well enough justified and too non-generic to be a good explanation of the observed phenomenology. Everything points to the fact that the center-of-mass fluctuations of the Eden model result from a strong violation of the Family-Vicsek scaling. As we may see from Eq. (115), this sort of violation implies stronger center-of-mass fluctuations. This point is discussed further in the next section.

In summary, we can say that the result $C_{m} \sim t^{2 / 5}$ suggests a strong violation of the Family-Vicsek scaling by the surface fluctuations of the $(1+1)$-dimensional Eden model. Although the linear law $C_{m} \sim t^{(1-\gamma d) / 2}$ does not reproduce the results quantitatively, we still expect from it a qualitative description of the dynamics, as the strong decrease in this exponent was already reported in $(2+1)$ dimensions. According to the linear law, the center-of-mass fluctuations 
should decrease for increasing growth velocity and spatial dimension. Note also that the nonlinearity seems to be a necessary ingredient; the linearization of the KPZ equation proposed in [12] reads, in Fourier space,

$$
\frac{d}{d t}\left\langle h_{n}^{2}\right\rangle=-A|n|^{3 / 2}\left\langle h_{n}^{2}\right\rangle+\frac{B}{|n|^{1 / 2}},
$$

for some constants $A$ and $B$ and in the case of a nongrowing domain. This equation supports unbounded fluctuations as revealed by the divergent stochastic contribution in the limit $n \rightarrow 0$, and so this does not constitute a good model for predicting the center-of-mass fluctuations.

\section{APPLICATIONS TO THE EDEN MODEL}

In statistical mechanics it has been customary to classify the behavior of discrete models within universality classes defined by continuum field theories. Nonequilibrium growth theories have been, by no means, an exception to this rule $[49,50]$. In this sense, one would be interested in finding the universality class the Eden model belongs to. According to the simulations performed in the planar geometry the Eden model belongs to the KPZ universality class [1]. This agrees with the measured exponent $\beta=1 / 3$ in radial systems [4]. However, as we have already seen, there are at least two possible universality classes associated with the KPZ equation in radial systems: dilution-KPZ and dilatation-KPZ. The first one is characterized by a behavior more akin to that of planar systems, and the second one by memory effects which imply a departure from the Family-Vicsek scaling. According to the measurement of the autocorrelation exponent of the Eden model in [12], which yielded $\lambda=1 / 3$, the Eden model would be in the dilatation-KPZ universality class (one would expect $\lambda=4 / 3$ for dilution dynamics according to the theory developed hereand in [17]). This fact admits a simple explanation. In the Eden model, cells are aggregated to the colony peripherally in such a way that the positions of cells already present are not modified. Consequently, as the system grows, no dilution is redistributing its constituents. So the rigidity of the Eden model may well be at the origin of the memory effects present at its interface [12], which presumably place it in the dilatation-KPZ universality class. But to be sure, one would still need, of course, to verify that this implies no contradiction with the center-of-mass fluctuations as discussed in Sec. VIII.

As we have already mentioned, the Eden model may be thought of as an idealization of a developing cell colony. Of course, as was completely clear from the very beginning [2,3], there are multiple factors of a biological, chemical, and even physical nature that are not captured by this model. Apart from these, one could be interested in improving the model in purely statistical mechanical terms. To this end, one may look for inspiration to real cell colonies. The structure of a rapidly developing cell colony would be dominated by dilution effects, originating in the birth of new cells whose volume causes the displacement of existing cells. This feature is not captured by any sort of Eden model (diverse proliferation rules, on- or off-lattice, etc.) and is fundamental in preserving the Family-Vicsek scaling, as we have already seen. So it seems quite reasonable to modify the Eden model in order to remove its rigidity, allowing bulk cell proliferation and the displacement of existing cells, both in the bulk and at the interface, by the newborn cells. This would be interesting not just in modeling terms, but also for introducing dilution in the model and, consequently, shifting its universality class.

\section{CONCLUSIONS AND OUTLOOK}

In this work we have investigated the role of dilution and decorrelation on radial growth. Dilution drives matter redistribution along the growing interface: as the surface becomes larger the already deposited matter occupies a smaller fraction of the interface, which is being simultaneously complemented with incoming matter, the actual driving force of domain growth in radial systems. Dilution is important for any rate of domain growth, as it keeps the interfacial density constant, but especially for rapidly growing domains, for which the diffusion mechanism becomes irrelevant and dilution becomes the sole responsible for the propagation of correlations on the macroscopic scale. The importance of dilution is such that in its absence, which takes place in the alternative dilatation dynamics, strong memory effects arise. These include an enhanced stochasticity, which separates the behavior of the large spatial scale limit of the two-points correlation function from that dictated by the Family-Vicsek scaling, and the appearance of nonuniversal critical exponents in the marginally rough regime, characterized by the equality $\zeta=d$. As have seen, both universality and the Family-Vicsek structure of the correlation function are recovered by virtue of dilution. This, at least, is what happens in the cases mostly considered here, which focus on unveiling the effects of domain growth. Topological effects are indeed present when one considers global scaling properties of hyperspherical interfaces [20].

As dilution propagates correlations at the same speed at which the interface grows, a global correlation becomes impossible for fast domain growth. This leads to decorrelation or, in other words, to a whitening of the interfacial profile in the sense that distant points become uncorrelated. Decorrelation may be macroscopic, which is evident only if we regard the dynamics from a spatial scale of the same order of magnitude as the system size, or microscopic, in which case it is apparent for much smaller length scales. Microscopic decorrelation supports rapid roughening, i.e., growth regimes characterized by $\beta>1 / 2$. These appear naturally in the context of radial growth, e.g., by considering the IS equation, which results from a geometric variational principle and for which $\zeta=d=2$ and $\delta=3$, and thus it shows rapid roughening for all $\gamma>1$. A consequence of macroscopic decorrelation is the advent of a scale-dependent interfacial fractal dimension (so the surface becomes a scale-dependent fractal) which we have conjectured to be self-similar.

There are several theoretical problems that can be straightforwardly analyzed with the techniques introduced here. We have, e.g., considered radial interfaces whose mean radius grows as a power law of time $\langle r\rangle \sim t^{\gamma}$. This result has been obtained by means of a linear mechanism in which an explicit power-law dependence on time has been considered; see Eq. (10). This linear mechanism can be 
substituted by a nonlinear one in which time does not appear explicitly,

$$
\partial_{t} r=\gamma F^{1 / \gamma} r^{1-1 / \gamma}+\frac{1}{r^{d / 2} J(\vec{\theta})^{1 / 2}} \xi(\theta, t),
$$

which yields, at the deterministic order, $R=F t^{\gamma}$ again, but it is the source at the first stochastic order of a term (reminiscent of dilution), which may be either stabilizing or destabilizing depending on the value of $\gamma$,

$$
\partial_{t} \rho=\frac{\gamma-1}{t} \rho+\frac{1}{F^{d / 2} t^{\gamma d / 2} J(\vec{\theta})^{1 / 2}} \eta(\theta, t) ;
$$

for small values of $\gamma$ the previous sections results are recovered, while for large values of $\gamma$, memory effects and enhanced (power law) stochasticity appear (which are standard effects of instability, as we have already seen), with the threshold value of $\gamma$ depending of whether or not we introduce dilution (in this concrete example dilution completely erases instability). Also, this instability mechanism, contrary to the ones studied here and in [16], which make the zeroth mode unstable and the $l=1$ ones marginal, is able to destabilize all modes. Different nonlinearities which might destabilize a fixed number of modes lying before some given $l^{*} \in \mathbb{N}$ can be easily devised too (basically by introducing terms of the form $-r^{-m}$ for some suitable $m \in \mathbb{N}$ in the corresponding equation of motion) and can even be cast on some geometric variational formulation as the cases considered in [16]. Of course, deciding which model is the good one must rely on numerical or experimental evidence based on the study of specific models or systems of interest.

As mentioned in Sec. I, part of the motivation for studying radial growth models such as the Eden and other models lies in the possible similarity of these to some forms of biological development, such as, e.g., cell colonies. The results of our study can be translated into this context to obtain some simple conclusions, provided the modeling assumptions make sense for some biological system. The structure of a rapidly developing cell colony would be dominated by dilution effects, originating in the birth of new cells whose volume causes the displacement of the existent cells. If the rate of growth is large enough, this motion will dominate over any possible random dispersal of the individual cells. It is remarkable that such a consequence simply appears by considering domain growth, and it is not necessary to introduce corrections coming from the finite size of the constituents. This is the dilution-dominated situation we have formalized by means of the (decorrelation) inequality $\gamma>1 / \zeta$ (assuming in this case $\delta=\zeta$ ). In this case the overall appearance of the colony would be macroscopically isotropic (i.e., disregarding microscopic details). If we were to introduce some control protocol in order to break this symmetry, we would need to eliminate colony constituents (possibly randomly selected) at a rate high enough that the effective growth velocity would be one that reversed the decorrelation inequality. This would make diffusion, instead of system size growth, the dominant mechanism responsible for the colony macroscopic structure. Consequently, the macroscopic isotropy would be lost by means of diffusion-mediated anisotropic fluctuations developing on large scales. This can be considered as a purely stochastic instability which could perhaps be connected to the early stages of some mechanism of biological pattern formation. For the one-dimensional Eden model, accepting that it belongs to the KPZ universality class, one finds $\gamma=1$ and $z=3 / 2$. If $z$ played the same role for the nonlinear KPZ equation as $\zeta$ for the linear equations considered here (as it is reasonable to expect), the Eden model would be in the uncorrelated regime. In order to control it we would need to eliminate its cells at a rate such that the effective growth rate obeyed $\gamma<2 / 3$. For the two-dimensional Eden model, if its behavior were still analogous to that of the KPZ equation, we would find $z>3 / 2$ and thus greater difficulty in system control. Note that for the particular growth rules of the Eden model, one would need to eliminate peripheral cells in order to control the system. This would not be so in the case of an actual bacterial colony, for which bulk cells are still able to reproduce, and so cell elimination could be performed randomly across the whole colony. Of course, these conclusions are speculative as long as radial growth equations are not proved to reasonably model some biological system.

In more general terms, we have found that the surface fluctuations of the Eden model presumably strongly violate the Family-Vicsek scaling. We have identified the absence of dilution in this model as the reason underlying this violation. In that sense, this model would not be able to describe growing cell colonies, precisely because it assumes spurious rigidity of bulk cells. On the other hand, it would be better suited to describe the radial growth of crystalline structures [51]. We have also found that reparametrization invariance as defined in [22] implicitly implies dilatation dynamics. Our results call for an extension of the generalization of Langevin dynamics to arbitrary geometries in order to capture both dilution and dilatation scenarios and the associated bifurcation of universality classes. This same remark would affect equilibrium systems as well, but in this case, of course, the domain evolution will drive them out of equilibrium, unless growth is quasistatic [52].

\section{ACKNOWLEDGMENT}

This work was partially supported by the MICINN (Spain) through Project No. MTM2010-18128.

\section{APPENDIX: HIGHER ORDER PERTURBATION EXPANSION}

As we mentioned in Sec. II, the first-order correction in the small noise expansion is a Gaussian stochastic process. We try to go beyond this order in this Appendix, and we show the difficulties that arise in trying to do so. We again focus on the radial random deposition equation (10), and assume the solution form

$$
r(\vec{\theta}, t)=R(t)+\sqrt{\epsilon} \rho(\vec{\theta}, t)+\epsilon \rho_{2}(\vec{\theta}, t),
$$

where the noise intensity $\epsilon$ will be used as the small parameter [23]. Substituting this solution form into Eq. (10) we obtain the equation hierarchy

$$
\begin{gathered}
\partial_{t} R=F \gamma t^{\gamma-1}, \\
\partial_{t} \rho_{1}=\frac{1}{F^{d / 2} t^{\gamma d / 2}} \frac{\eta(\vec{\theta}, t)}{J(\vec{\theta})^{1 / 2}},
\end{gathered}
$$




$$
\partial_{t} \rho_{2}=-\frac{d}{2 F^{1+d / 2}} \frac{\rho_{1}}{t^{\gamma+d \gamma / 2}} \frac{\eta(\vec{\theta}, t)}{J(\vec{\theta})},
$$

where $\xi=\sqrt{\epsilon} \eta$ and both $\eta$ and $\xi$ are now zero-mean quasiwhite Gaussian processes whose correlations are given by

$$
\begin{gathered}
\langle\eta(\vec{\theta}, t) \eta(\vec{\theta}, t)\rangle=C\left(\vec{\theta}-\vec{\theta}^{\prime}\right) \delta\left(t-t^{\prime}\right), \\
\langle\xi(\vec{\theta}, t) \xi(\vec{\theta}, t)\rangle=\epsilon C\left(\vec{\theta}-\vec{\theta}^{\prime}\right) \delta\left(t-t^{\prime}\right),
\end{gathered}
$$

where $C(\cdot)$ is some regular function approximating the Dirac delta; the necessity for the quasiwhite assumption will we clear in few lines. These equations have been derived assuming $\sqrt{\epsilon} \ll F t^{\gamma}$, and we will further assume a zero value for both initial perturbations as in Sec. II. The solution to the first two was characterized in Sec. II, where the approximating function $C(\cdot)$ was substituted by the Dirac $\delta$. Here $R$ is a deterministic function and $\rho_{1}$ is a zero-mean Gaussian stochastic process that is completely determined by its correlation function. The stochastic function $\rho_{2}$ is a zero-mean process too, but it is not Gaussian this time, and its correlation (which no longer completely determines the process) is given by

$$
\left\langle\rho_{2}(\vec{\theta}, t) \rho_{2}\left(\vec{\theta}^{\prime}, s\right)\right\rangle=\frac{d^{2}}{4 F^{2+2 d}(1-\gamma d)}\left[\frac{(\min \{t, s\})^{2-2 \gamma-2 \gamma d}-t_{0}^{2-2 \gamma-2 \gamma d}}{2-2 \gamma-2 \gamma d}-t_{0}^{1-\gamma d} \frac{(\min \{t, s\})^{1-2 \gamma-\gamma d}-t_{0}^{1-2 \gamma-\gamma d}}{1-2 \gamma-\gamma d}\right] \frac{C\left(\vec{\theta}-\vec{\theta}^{\prime}\right)^{2}}{J(\vec{\theta}) J\left(\vec{\theta}^{\prime}\right)},
$$

if $\gamma d \neq 1, \gamma(1+d) \neq 1$, and $\gamma(2+d) \neq 1$. If $\gamma d=1$, we find

$$
\left\langle\rho_{2}(\vec{\theta}, t) \rho_{2}\left(\vec{\theta}^{\prime}, s\right)\right\rangle=\frac{1}{16 F^{2+2 d} \gamma^{4}}\left\{t_{0}^{-2 \gamma}-[\min \{t, s\}]^{-2 \gamma}\left[1+2 \gamma \ln \left(\frac{\min \{t, s\}}{t_{0}}\right)\right]\right\} \frac{C\left(\vec{\theta}-\vec{\theta}^{\prime}\right)^{2}}{J(\vec{\theta}) J\left(\vec{\theta}^{\prime}\right)}
$$

if $\gamma(1+d)=1$, then

$$
\left\langle\rho_{2}(\vec{\theta}, t) \rho_{2}\left(\vec{\theta}^{\prime}, s\right)\right\rangle=\frac{d^{2}}{4 F^{2+2 d} \gamma}\left[\ln \left(\frac{\min \{t, s\}}{t_{0}}\right)+\frac{t_{0}^{\gamma}}{\gamma}\left([\min \{t, s\}]^{-\gamma}-t_{0}^{-\gamma}\right)\right] \frac{C\left(\vec{\theta}-\vec{\theta}^{\prime}\right)^{2}}{J(\vec{\theta}) J\left(\vec{\theta}^{\prime}\right)} ;
$$

and if $\gamma(2+d)=1$, we get

$$
\left\langle\rho_{2}(\vec{\theta}, t) \rho_{2}\left(\vec{\theta}^{\prime}, s\right)\right\rangle=\frac{d^{2}}{8 F^{2+2 d} \gamma}\left[\frac{(\min \{t, s\})^{2 \gamma}-t_{0}^{2 \gamma}}{2 \gamma}-t_{0}^{2 \gamma} \ln \left(\frac{\min \{t, s\}}{t_{0}}\right)\right] \frac{C\left(\vec{\theta}-\vec{\theta}^{\prime}\right)^{2}}{J(\vec{\theta}) J\left(\vec{\theta}^{\prime}\right)} .
$$

The long-time behavior of the correlations, given by the condition $t, s \gg t_{0}$, is specified by the following two-times and one-time functions:

$$
\begin{gathered}
\left\langle\rho_{2}(\vec{\theta}, t) \rho_{2}\left(\vec{\theta}^{\prime}, s\right)\right\rangle=\frac{d^{2}}{4 F^{2+2 d}(1-\gamma d)} \frac{(\min \{t, s\})^{2-2 \gamma-2 \gamma d}}{2-2 \gamma-2 \gamma d} \frac{C\left(\vec{\theta}-\vec{\theta}^{\prime}\right)^{2}}{J(\vec{\theta}) J\left(\vec{\theta}^{\prime}\right)}, \\
\left\langle\rho_{2}(\vec{\theta}, t) \rho_{2}\left(\vec{\theta}^{\prime}, t\right)\right\rangle=\frac{d^{2}}{4 F^{2+2 d}(1-\gamma d)} \frac{t^{2-2 \gamma-2 \gamma d}}{2-2 \gamma-2 \gamma d} \frac{C\left(\vec{\theta}-\vec{\theta}^{\prime}\right)^{2}}{J(\vec{\theta}) J\left(\vec{\theta}^{\prime}\right)}
\end{gathered}
$$

when $\gamma(d+1)<1$; if $\gamma(d+1)=1$, then

$$
\begin{gathered}
\left\langle\rho_{2}(\vec{\theta}, t) \rho_{2}\left(\vec{\theta}^{\prime}, s\right)\right\rangle=\frac{d^{2}}{4 F^{2+2 d} \gamma} \ln (\min \{t, s\}) \frac{C\left(\vec{\theta}-\vec{\theta}^{\prime}\right)^{2}}{J(\vec{\theta}) J\left(\vec{\theta}^{\prime}\right)} \\
\left\langle\rho_{2}(\vec{\theta}, t) \rho_{2}\left(\vec{\theta}^{\prime}, t\right)\right\rangle=\frac{d^{2}}{4 F^{2+2 d} \gamma} \ln (t) \frac{C\left(\vec{\theta}-\vec{\theta}^{\prime}\right)^{2}}{J(\vec{\theta}) J\left(\vec{\theta}^{\prime}\right)} ;
\end{gathered}
$$

and, finally, when $\gamma(d+1)>1$, we find

$$
\left\langle\rho_{2}(\vec{\theta}, t) \rho_{2}\left(\vec{\theta}^{\prime}, s\right)\right\rangle=\frac{d^{2}}{8 F^{2+2 d}} \frac{t_{0}^{2-2 \gamma-2 \gamma d}}{1-(3+2 d) \gamma+\left(2+3 d+d^{2}\right) \gamma^{2}} \frac{C\left(\vec{\theta}-\vec{\theta}^{\prime}\right)^{2}}{J(\vec{\theta}) J\left(\vec{\theta}^{\prime}\right)},
$$


a correlation function that vanishes in the limit $t_{0} \rightarrow \infty$. Now it is clear why we needed the quasiwhite approximation: for a regular function $C(\cdot)$ the expression $C(\cdot)^{2}$ makes sense, contrary to what happens if we substitute it with the Dirac $\delta$ to get $\delta(\cdot)^{2}$. This is the first indication of the failure of the higher order perturbation theory.

We now examine the effect that dilution has on the random function $\rho_{2}$, which in this case obeys the equation

$$
\partial_{t} \rho_{2}=-\frac{\gamma d}{t} \rho_{2}-\frac{d}{2} \frac{(d+1)^{1+d / 2}}{F^{1+d / 2} t^{\gamma+\gamma d / 2}} \frac{\rho_{1}(\vec{\theta}, t) \xi(\vec{\theta}, t)}{J(\vec{\theta})} .
$$

In this case the long-time correlation function reads

$$
\begin{aligned}
\left\langle\rho_{2}(\vec{\theta}, t) \rho_{2}\left(\vec{\theta}^{\prime}, s\right)\right\rangle= & \frac{d^{2}(d+1)^{2+2 d}}{8 F^{2+2 d}(\gamma d+1)(1-\gamma)}(t s)^{-\gamma d} \\
& \times \min \{t, s\}^{2-2 \gamma} \frac{C\left(\vec{\theta}-\vec{\theta}^{\prime}\right)^{2}}{J(\vec{\theta}) J\left(\vec{\theta}^{\prime}\right)}
\end{aligned}
$$

if $\gamma<1$,

$$
\begin{aligned}
\left\langle\rho_{2}(\vec{\theta}, t) \rho_{2}\left(\vec{\theta}^{\prime}, s\right)\right\rangle= & \frac{d^{2}(d+1)^{1+2 d}}{4 F^{2+2 d}}(t s)^{-d} \\
& \times \ln [\min \{t, s\}] \frac{C\left(\vec{\theta}-\vec{\theta}^{\prime}\right)^{2}}{J(\vec{\theta}) J\left(\vec{\theta}^{\prime}\right)}
\end{aligned}
$$

if $\gamma=1$, and

$$
\begin{aligned}
\left\langle\rho_{2}(\vec{\theta}, t) \rho_{2}\left(\vec{\theta}^{\prime}, s\right)\right\rangle= & \frac{d^{2}(d+1)^{2+2 d}}{8 F^{2+2 d}(\gamma d+1)(\gamma-1)}(t s)^{-\gamma d} t_{0}^{2-2 \gamma} \\
& \times \frac{C\left(\vec{\theta}-\vec{\theta}^{\prime}\right)^{2}}{J(\vec{\theta}) J\left(\vec{\theta}^{\prime}\right)}
\end{aligned}
$$

if $\gamma>1$. The one-time correlation function is then

$$
\begin{aligned}
\left\langle\rho_{2}(\vec{\ell}, t) \rho_{2}\left(\vec{\ell}^{\prime}, t\right)\right\rangle= & \frac{d^{2}(d+1)^{2+2 d}}{8 F^{2+2 d}(\gamma d+1)(1-\gamma)} t^{2-2 \gamma} \\
& \times \frac{C\left(\vec{\ell}-\vec{\ell}^{\prime}\right)^{2}}{J\left(t^{-\gamma} \vec{\ell}\right) J\left(t^{-\gamma} \vec{\ell}^{\prime}\right)}
\end{aligned}
$$

$$
\begin{aligned}
& \text { if } \gamma<1, \\
& \qquad\left\langle\rho_{2}(\vec{\ell}, t) \rho_{2}\left(\vec{\ell}^{\prime}, t\right)\right\rangle=\frac{d^{2}(d+1)^{1+2 d}}{4 F^{2+2 d}} \ln (t) \frac{C\left(\vec{\ell}-\vec{\ell}^{\prime}\right)^{2}}{J\left(t^{-\gamma} \vec{\ell}\right) J\left(t^{-\gamma} \vec{\ell}^{\prime}\right)}
\end{aligned}
$$

if $\gamma=1$, and

$$
\begin{aligned}
\left\langle\rho_{2}(\vec{\ell}, t) \rho_{2}\left(\vec{\ell}^{\prime}, t\right)\right\rangle= & \frac{d^{2}(d+1)^{2+2 d}}{8 F^{2+2 d}(\gamma d+1)(\gamma-1)} t_{0}^{2-2 \gamma} \\
& \times \frac{C\left(\vec{\ell}-\vec{\ell}^{\prime}\right)^{2}}{J\left(t^{-\gamma} \vec{\ell}\right) J\left(t^{-\gamma} \vec{\ell}^{\prime}\right)}
\end{aligned}
$$

if $\gamma>1$, where $\vec{\ell}-\vec{\ell}^{\prime}=t^{\gamma}\left(\vec{\theta}-\vec{\theta}^{\prime}\right), C\left(\vec{\ell}-\vec{\ell}^{\prime}\right)=t^{-\gamma d} C(\vec{\theta}-$ $\left.\vec{\theta}^{\prime}\right)$, and we have assumed that the approximating function $C(\cdot)$ has the same homogeneity as the Dirac $\delta$. Although it is evident that dilution carries out a measurable action, particularly erasing part of the memory effects, the result is far from satisfactory. In all cases the prefactor deviates from the expected random deposition form $t^{2}$ [53], the unexpected critical value $\gamma=1$ has appeared, and for $\gamma \geqslant 1$ memory effects are present as signaled by the logarithm and the $t_{0}$ dependence, respectively; and the situation is further complicated by the presence of the factor $C(\cdot)^{2}$, which becomes singular in the white-noise limit. All of these elements suggest the failure of the small noise expansion beyond the first order. Classical results suggest the possibility of constructing a systematic approach to the solution of some nonlinear stochastic differential equations by continuing the small noise expansion to higher orders [23]. Our present results suggest the failure of this sort of expansion beyond the Gaussian (which turns out to be the first) order in very much the same way that the Kramers-Moyal expansion of the master equation [54] and the Chapman-Enskog expansion of the Boltzmann equation [55] fail beyond the Fokker-Planck and Navier-Stokes orders, respectively.
[1] A.-L. Barabási and H. E. Stanley, Fractal Concepts in Surface Growth (Cambridge University Press, Cambridge, 1995).

[2] M. Eden, in Symposium on Information Theory in Biology, edited by H. P. Yockey (Pergamon Press, New York, 1958).

[3] M. Eden, in Proceedings of the Fourth Berkeley Symposium on Mathematical Statistics and Probability, edited by J. Neyman (University of California Press, Berkeley, 1961).

[4] S. C. Ferreira Jr. and S. G. Alves, J. Stat. Mech. (2006) P11007.

[5] T. A. Witten Jr. and L. M. Sander, Phys. Rev. Lett. 47, 1400 (1981).

[6] J. M. Hammersley and G. Mazzarino, Comb. Probab. Comput. 3, 471 (1994).

[7] H. Hinrichsen, Adv. Phys. 49, 815 (2000).

[8] J. Cardy, Ann. Phys. 318, 81 (2005).

[9] F. Family and T. Vicsek, J. Phys. A 18, L75 (1985).

[10] R. Kapral, R. Livi, G.-L. Oppo, and A. Politi, Phys. Rev. E 49, 2009 (1994).
[11] M. T. Batchelor, B. I. Henry, and S. D. Watts, Physica A 260, 11 (1998).

[12] S. B. Singha, J. Stat. Mech. (2005) P08006.

[13] C. Escudero, Phys. Rev. E 73, 020902(R) (2006).

[14] C. Escudero, Phys. Rev. E 74, 021901 (2006).

[15] C. Escudero, Phys. Rev. Lett. 100, 116101 (2008).

[16] C. Escudero, Ann. Phys. 324, 1796 (2009).

[17] C. Escudero, J. Stat. Mech. (2009) P07020.

[18] E. J. Crampin, E. A. Gaffney, and P. K. Maini, Bull. Math. Biol. 61, 1093 (1999).

[19] C. Escudero, e-print arXiv:0907.0898v1 [cond-mat.stat-mech].

[20] C. Escudero, e-print arXiv:1001.3323v2 [cond-mat.stat-mech].

[21] A. Maritan, F. Toigo, J. Koplik, and J. R. Banavar, Phys. Rev. Lett. 69, 3193 (1992).

[22] M. Marsili, A. Maritan, F. Toigo, and J. R. Banavar, Rev. Mod. Phys. 68, 963 (1996).

[23] C. W. Gardiner, Handbook of Stochastic Methods (SpringerVerlag, Berlin, 1996). 
[24] M. Abramowitz and I. A. Stegun, Handbook of Mathematical Functions with Formulas, Graphs, and Mathematical Tables (Dover, New York, 1964).

[25] J. M. Pastor and J. Galeano, Central European J. Phys. 5(4), 539 (2007).

[26] C. Escudero, Phys. Rev. Lett. 101, 196102 (2008).

[27] C. Escudero and E. Korutcheva, e-print arXiv:1004.2725.

[28] Z. Wen and J. Avery, J. Math. Phys. 26, 396 (1985).

[29] E. Hernández-García, T. Ala Nissila, and M. Grant, Europhys. Lett. 21, 401 (1993).

[30] M. Kardar, G. Parisi, and Y.-C. Zhang, Phys. Rev. Lett. 56, 889 (1986).

[31] H. S. Wio, Int. J. Bif. Chaos 19, 2813 (2009).

[32] M. Kardar, Nucl. Phys. B 290, 582 (1987).

[33] M. Lässig, Nucl. Phys. B 448, 559 (1995).

[34] D. Forster, D. R. Nelson, and M. J. Stephen, Phys. Rev. A 16, 732 (1977).

[35] E. Medina, T. Hwa, M. Kardar, and Y.-C. Zhang, Phys. Rev. A 39, 3053 (1989).

[36] D. S. Jones and B. D. Sleeman, Differential Equations and Mathematical Biology (CRC Press, London, 2003).

[37] A. Berera and D. Hochberg, Phys. Rev. Lett. 99, 254501 (2007).

[38] A. Berera and D. Hochberg, Nucl. Phys. B 814, 522 (2009).

[39] H. S. Wio, J. A. Revelli, R. R. Deza, C. Escudero, and M. S. de La Lama, Europhys. Lett. 89, 40008 (2010).

[40] H. S. Wio, J. A. Revelli, R. R. Deza, C. Escudero, and M. S. de La Lama, Phys. Rev. E 81, 066706 (2010).

[41] H. S. Wio, C. Escudero, J. A. Revelli, R. R. Deza, and M. S. de La Lama, Philos. Trans. R. Soc. A 369, 396 (2011).
[42] M. Henkel, Nucl. Phys. B 641, 405 (2002).

[43] A. Rothlein, F. Baumann, and M. Pleimling, Phys. Rev. E 74, 061604 (2006).

[44] H. C. Fogedby, Phys. Rev. Lett. 94, 195702 (2005).

[45] H. C. Fogedby, Phys. Rev. E 73, 031104 (2006).

[46] E. W. Kuennen and C. Y. Wang, J. Stat. Mech. (2008) P05014.

[47] J. Villain, J. Phys. I (France) 1, 19 (1991).

[48] Z.-W. Lai and S. Das Sarma, Phys. Rev. Lett. 66, 2348 (1991).

[49] C. A. Haselwandter and D. D. Vvedensky, Phys. Rev. Lett. 98, 046102 (2007).

[50] C. A. Haselwandter and D. D. Vvedensky, Phys. Rev. E 76, 041115 (2007).

[51] S. V. Khare and T. L. Einstein, Phys. Rev. B 54, 11752 (1996).

[52] H. G. Ballesteros, L. A. Fernández, V. Martín-Mayor, A. Muñoz Sudupe, G. Parisi, and J. J. Ruiz-Lorenzo, Nucl. Phys. B 512, 681 (1998).

[53] This prefactor always shows a smaller exponent, and the expected $t^{2}$ only appears in the limit $\gamma \rightarrow 0$. This reduction of the prefactor exponent happens equally in the presence and the absence of dilution and is the opposite trend from the one observed in the dilution-free Gaussian order, which tends to increase this exponent $[17,19]$. All this strongly suggests the failure of the perturbation theory at this order, rather than an effect related to dilution or dilatation.

[54] R. F. Pawula, Phys. Rev. 162, 186 (1967).

[55] C. Cercignani, The Boltzmann Equation and Its Applications (Springer-Verlag, New York, 1987). 